AperTO - Archivio Istituzionale Open Access dell'Università di Torino

\title{
Application of mass spectrometry to hair analysis for forensic toxicological investigations
}

\section{This is the author's manuscript}

Original Citation:

\section{Availability:}

This version is available http://hdl.handle.net/2318/117256

since 2015-12-29T11:15:33Z

Published version:

DOI:10.1002/mas.21364

Terms of use:

Open Access

Anyone can freely access the full text of works made available as "Open Access". Works made available under a Creative Commons license can be used according to the terms and conditions of said license. Use of all other works requires consent of the right holder (author or publisher) if not exempted from copyright protection by the applicable law. 


\title{
APPLICATION OF MASS SPECTROMETRY TO HAIR ANALYSIS FOR FORENSIC TOXICOLOGICAL INVESTIGATIONS
}

\author{
Marco Vincenti, ${ }^{1,2^{*}}$ Alberto Salomone, ${ }^{1}$ Enrico Gerace, ${ }^{1}$ and \\ Valentina Pirro ${ }^{1,2}$ \\ ${ }^{1}$ Centro Regionale Antidoping e di Tossicologia "A. Bertinaria", Orbassano (TO), Italy \\ ${ }^{2}$ Dipartimento di Chimica, Università di Torino, Torino, Italy
}

Running head title: "Hair Analysis for Forensic Toxicology"

Keywords: Hair, Toxicology, Drugs, Mass Spectrometry, Forensic

\begin{abstract}
The increasing role of hair analysis in forensic toxicological investigations principally owes to recent improvements of mass spectrometric instrumentation. Research achievements during the last six years in this distinctive application area of analytical toxicology are reviewed. The earlier state of the art of hair analysis was comprehensively covered by a dedicated book (Kintz, 2007a), that represents key reference of the present overview. Whereas the traditional organization of analytical methods in forensic toxicology divided target substances into quite homogeneous groups of drugs, with similar structures and chemical properties, the current approach often takes advantage of the rapid expansion of multiclass and multiresidue analytical procedures; the latter is made possible by the fast operation and extreme sensitivity of modern mass spectrometers. This change in the strategy of toxicological analysis is reflected in the presentation of the recent literature material, which is mostly based on a fit-for-purpose logic. Thus, general screening of unknown substances is applied in diverse forensic contexts than drugs of abuse testing, and different instrumentation (triple quadrupoles, time-of-flight analyzers, linear and orbital traps) is utilized to optimally cope with the scope. Other key issues of modern toxicology, such as cost reduction and high sample throughput, are discussed with reference to procedural and instrumental alternatives.
\end{abstract}

\section{*Correspondence to:}

Prof. Marco Vincenti,

Dipartimento di Chimica, Università di Torino,

Via Pietro Giuria 5, 10125 - Torino, Italy

Tel.: +390116705264

Fax: +390116705242

E-mail: marco.vincenti@unito.it 


\section{INTRODUCTION}

2

The analysis of biological specimens to detect of various types of drugs is consistently requested within forensic investigations as a necessary step to prove exposure to these substances. Although blood and urine remain the matrices of choice to detect recent exposure, hair analysis is gaining ever-increasing importance, as its potentials, limitations, and new application areas are progressively uncovered. Although, for most drugs, any evidence of exposure is erased from blood and urine a few days (or hours) after their intake because of the natural metabolic and excretion body processes, a minute portion of the same drugs and metabolites is ultimately incorporated through several routes (blood capillaries, sweat, and sebum) into the keratin structure of the hair, from which it is hardly removed. This drug-fixing into the hair structure resists hair growth for several months and leads to a potential chronological trace of drug exposure, with farther periods corresponding to the hair segments more distant from the hair root. The continuous improvement of analytical procedures and instrumental technologies allows one to determine the very small amounts of drugs included into the hair at ever-decreasing concentrations, insomuch as there are currently several scientific reporting of drug detection in hair after a single exposure. Progresses in chromatographic and mass spectrometric techniques have both equally contributed to the impressive results achievable nowadays with modern forensic toxicology in hair analysis.

On the negative side of hair analysis, there are several potential sources of bias that should always be taken into account in order to produce reliable conclusions from experimental results. First of all, hair is not a homogeneous matrix; thus, careful and representative hair sampling is a necessary prerequisite of correct analysis. Secondly, the degree of hair incorporation depends on the chemical structure and related properties of the drug, including melanin affinity, lipophilicity and membrane permeability (Kintz, 2012a). Third, adsorption of external substances from the environment is occasionally claimed as a source of false-positive results, especially for professionally-exposed subjects and volatile substances, such as, for example, cocaine (LeBeau \& Montgomery, 2009). To prevent this inconvenience, preliminary washing of hair samples is always recommended to eliminate any possible interfering substances adsorbed onto the external surface of the hair. Segmental analysis and acceptance of cut-off values often rule out the chance that exogenous drug sources might have contaminated the internal keratin structure. Forth, strong cosmetic hair treatments, such as the use of oxidants or highly basic coloring, might damage the keratin structure to favor release of incorporated substances to finally lead to false-negative results. Also, repeated shampooing might partly wash out the hydrophilic drugs incorporated into the hair. 
Lastly, the use of cosmetic products such as grease, sprays, and gels might also interfere with hair analysis.

A comprehensive review of drug testing in hair was published in a dedicated book edited by Pascal Kintz (2007a). Since then, only one review was published on abused drug analysis in hair samples, in which chapters are organized on the basis of the various classes of substances (Wada et al., 2010). The present review updates Kintz's book, under the perspective of its chapters 10-15 (Kintz, 2007a); namely, under application areas point of view. The studies published before 2006 are only incidentally cited in the present review, whereas extensive coverage of the papers published in the last six years is presented.

\section{APPLICATION AREAS OF MASS SPECTROMETRY TO HAIR ANALYSIS IN FORENSIC TOXICOLOGY}

\section{A. Targeted Drug Screening for Specific Drug Classes}

Although screening of psychoactive substances in biological specimens might have a variety of objectives, in terms of target chemical classes and purposes of control, legislation of most countries make a clear distinction between drugs of abuse, whose use is prohibited in almost all circumstances, and pharmaceutical substances, whose use is permitted under medical control and prescription, even though they are occasionally abused. Most routine screening, such as, for example, workplace testing, are addressed to the drugs of abuse only, whereas other psychoactive pharmaceuticals are searched in selected subjects and follow anamnestic indications. In particular, detection of the most-common drugs of abuse in hair samples (Kintz, 2007a; Kintz et al., 2006; Pragst \& Balikova, 2006; Wada et al., 2010) is increasingly requested for the retrospective withdrawal control of habitual drug abusers, as well as in other toxicological investigations, such as workplace drug testing [EWDTS (European Workplace Drug Testing Society), 2010; Caplan \& Goldberger, 2001], driving re-licensing, occasional or pre-natal exposure to drugs (Kintz, 2007b; Falcon et al., 2012), and post-mortem toxicology. Therefore, although hair samples are routinely collected and analyzed in forensic and toxicological laboratories, most published methods were implemented to determine class-specific groups of compounds, mainly cannabinoids, amphetamines, cocaine, and opiates, even though the analytical procedures have been constantly updated (Kintz, 2007a; Pragst \& Balikova, 2006; Wada et al., 2010). 


\section{Classes of drugs}

Although the widespread abuse of hashish or marijuana makes the detection of long-term exposure to $\Delta^{9}$-tetrahydrocannabinol (THC) by hair analysis extremely important in clinical and forensic contexts, drug inhalation and adsorption from environmental smoke are frequently claimed to justify positive detection of THC and/or its main metabolite. Although a cut-off of $0.1 \mathrm{ng} / \mathrm{mg}$ was traditionally recommended, more recently a cut-off of $0.05 \mathrm{ng} / \mathrm{mg}$ has been proposed as a suitable level to detect also occasional users (Pragst \& Nadulski, 2005) and for confirmation analyses (Cooper et al., 2012).

GC/MS is the technique of choice for THC detection in the majority of published methods, and no particular analytical improvement has been proposed recently, except for the increasing role played by headspace solid-phase microextraction (HS-SPME) procedures. Nadulski and Pragst developed, validated, and routinely applied to driving-ability examination a new method for cannabidiol (CBD) and cannabinol (CBN) with improved sensitivity (Nadulski \& Pragst, 2007). The authors used alkaline digestion of 15-30 mg hair aliquots, subsequent liquid-liquid extraction (LLE), automated HS-SPME after in-sample silylation, and GC/MS-SIM (selected ion monitoring) analysis. For THC, a limit of detection (LOD) of $0.012 \mathrm{ng} / \mathrm{mg}$ was obtained. A similar procedure without derivatization with $10 \mathrm{mg}$ of hair sample was proposed (Rodrigues de Oliveira et al., 2007), but the reported LOD and LOQ (limit of quantification) values of $0.07 \mathrm{ng} / \mathrm{mg}$ and $0.12 \mathrm{ng} / \mathrm{mg}$, respectively, are above the recommended cut-off limits and make the method apparently not appropriate for routine analysis. Emídio et al. developed and validated a method for the determination of THC, CBD, and CBN in hair samples, with HS-SPME combined with GC/MS/MS (ion trap) detection (Emídio et al., 2010). From a $10 \mathrm{mg}$ hair sample, an LOD of $0.031 \mathrm{ng} / \mathrm{mg}$ and LOQ of $0.062 \mathrm{ng} / \mathrm{mg}$ were obtained; the latter was slightly higher than the cut-off value of 0.05 ng/mg. Nonetheless, from 10 hair samples from cannabis users, an average concentration of 0.056 $\mathrm{ng} / \mathrm{mg}$ was found; i.e., below the reported LOQ.

Unlike THC, the improvement of existing analytical methods for stimulants, including cocaine and amphetamines, in hair samples has been widely investigated recently. Lee et al. used a standard GC/MS procedure, after derivatization with trifluoroacetic anhydride, in order to study the abundance ratio of methamphetamine (MA) and its metabolite amphetamine (AP) in hair and hence aid the positive results interpretation (Lee et al., 2009). High MA concentrations, together with low AP percentage, were related to severe and chronic drug abuse (Lee et al., 2009). In order to facilitate MA and AP analysis in hair, Miyaguchi et al. developed a simple and fast (1 hour) method for sample preparation named MiAMi (micropulverized extraction-acqueous acetylation- 
microextraction by packed sorbent) followed by GC/MS (Miyaguchi et al., 2009). The amount of hair sample required for qualitative analysis based on full-scan mass spectra was only $5 \mathrm{mg}$, whereas $1 \mathrm{mg}$ of a hair specimen was sufficient for amphetamine quantitation. The same group (Miyaguchi et al., 2007) previously published an extraction method on micropulverized hair for the HPLC-MS/MS determination of MA and AP, in which only $2 \mathrm{mg}$ of sample were used.

An LC-MS/MS method that used electrospray ionization (ESI) and a triple quadrupole instrument was developed and validated by Chèze et al. to determine AP, MA, 3,4methylenedioxyamphetamine (MDA), 3,4-methylenedioxymethamphetamine (MDMA, "ecstasy"), 3,4-methylenedioxyethamphetamine (MDEA), and N-methyl-1-(3,4-methylenedioxyphenyl)-2butanamine (MBDB) at low concentration levels, in hair, blood, and urine (Chèze et al., 2007). With $20 \mathrm{mg}$ of decontaminated hair, the experimental LODs ranged from $0.3 \mathrm{pg} / \mathrm{mg}$ for MBDB to $6.3 \mathrm{pg} / \mathrm{mg}$ for MDA, where the recommended cut-off limit for amphetamines is $200 \mathrm{pg} / \mathrm{mg}$ (Cooper et al., 2012). The same study reported a real forensic case, in which the high sensitivity of the LCESI-MS/MS method was exploited to detect MDMA in a hair sample, after a single oral dose administration to an unaware victim (Chèze et al., 2007). Another recent study reported the development of an LC-MS/MS method for the simultaneous determination of a large set of amphetamine-like anorectics and their metabolites in hair samples (Lee et. al., 2012). The procedure was used to analyze the diffusion of anorectics abuse in Korea. The hair incorporation of MA and AP following controlled administration of MA to seven volunteers was recently studied (Polettini et al., 2012). For these amphetamines, significant dependence (i.e.; linear direct correlation) of drug incorporation on hair melanin content was clearly demonstrated.

Besides MALDI-imaging techniques, several traditional methods were recently proposed to determine the most common stimulant drug (i.e., cocaine) in hair samples. The suggested cut-off concentration is $500 \mathrm{pg} / \mathrm{mg}$ for cocaine and $50 \mathrm{pg} / \mathrm{mg}$ for its main metabolite, benzoylecgonine (BZE) (Cooper et al., 2012). A GC/MS analytical method to quantify cocaine and its main metabolite BZE in hair samples was proposed (Barroso et al., 2008). Despite the simple instrumentation utilized, the method proved to be sensitive and specific, and allowed one to detect 20 and $15 \mathrm{pg} / \mathrm{mg}$ of cocaine and BZE, respectively, from only $20 \mathrm{mg}$ of sample.

Improved sensitivities were obtained with LC-MS/MS methods. Moore et al. developed and validated an LC-MS/MS procedure for the analysis of cocaine and its metabolites (BZE, cocaethylene, and norcocaine) in hair with an atmospheric pressure chemical ionization (APCI) source and a triple quadrupole (QqQ) mass analyzer (Moore et al., 2007). For all analytes, the LOQs (50 pg/mg) and LODs (25 pg/mg) made the method suitable for routine forensic analysis. 
A simple and fully validated procedure for the qualitative and quantitative determination of opiates in hair was presented (Barroso et al., 2010a). This paper describes a GC/MS method for the analysis of codeine, morphine, 6-monoacetylmorphine (6-MAM), 6-acetylcodeine, and tramadol in $20 \mathrm{mg}$ hair samples. The presence of the screened analytes was demonstrated in several real cases, among which it was proven that tramadol is occasionally abused by opiate addicts. A combination of opiates, cocaine and metabolites was simultaneously screened with an LC-ESI-MS/MS method (Huang et al., 2009). This method was fully validated and used in the analysis of 79 authentic hair samples, and demonstrated that a multiresidue approach might screen different classes of drugs at the same time.

The objective of cost reduction of workplace drug screening with a multiresidue UHPLCMS/MS strategy was explicitly cited in a recent study (Di Corcia et al., 2012). Thirteen analytes, including opiates, cocaine, amphetamines, THC, buprenorphine, methadone, and a few metabolites, were simultaneously screened in hair samples. A simple sample preparation combined with multiclass analysis and fast chromatographic separation allowed one to obtain high sample throughput, together with excellent sensitivity and selectivity; the procedure is valuable for large sample workload and reduced costs of analysis (Di Corcia et al., 2012).

\section{New instrumental set-up}

A new and original study, aimed to the direct detection of MA in hair samples, used imaging mass spectrometry (IMS) to perform micro-segmental analysis (Miki et al., 2011). In practice, a hair shaft was affixed to a carbon tape and manually cut lengthwise to produce micro-incisions at extremely close range with a razor and a microscope. After matrix deposition, IMS was obtained by matrix-assisted laser desorption ionization time-of-flight (MALDI-TOF) and MALDI-Fourier transform ion cyclotron resonance (FTICR) mass spectrometers. IMS produced a barcode-like image of methamphetamine on the longitudinal sections of the hair shafts, obtained from a methamphetamine chronic abuser, to indicate the periods and sequence of single MA intake (Miki et al., 2011). This innovative approach appears to be very promising in the forensic context, as soon as the hair sample preparation can be automated.

A MALDI hybrid triple quadrupole linear ion trap $\left(\mathrm{QqQ}_{\mathrm{LIT}}\right)$ instrument, equipped with a high repetition rate laser, was recently proposed to perform MS imaging on a single hair, for cocaine consumption monitoring (Porta et al., 2011). The QqQ LIT technology was exploited to simultaneously achieve target quantification with selected reaction monitoring (SRM) mode of operation and sensitive $\mathrm{MS}^{3}$ acquisition for confirmatory analysis. MS imaging of intact single hair 
sample offers much higher resolution than segmental LC-MS/MS analysis, and only depends on the distance between two adjacent laser shots. However, the minimal $0.1 \mathrm{~mm}$ resolution allowed by the laser and corresponding to a theoretical average hair growth of less than $12 \mathrm{~h}$, has currently little significance in forensic investigations, beacuse the complex nature of keratin incorporation mechanisms and the heterogeneous physiology of hair growth might provide a much larger bias on chronological assessment. Single washed hair samples were fixed onto a stainless steel MALDI plate with a double-sided adhesive foil, and the MALDI matrix was manually sprayed. First, an imaging profile of cocaine and metabolites was acquired in the SRM mode over the whole hair length, taking into account that highly selective transitions should be chosen to compensate for the absence of chromatographic separation. Secondly, MS/MS and $\mathrm{MS}^{3}$ experiments were performed for confirmatory identification. The entire analytical workflow is represented in Figure 1 (Porta et al., 2011). An experimental LOD of $5 \mathrm{ng} / \mathrm{mg}$ allows for cocaine detection in the hair of chronic abusers, but not after a single administration. Moreover, scaling down to single-hair analysis makes the choice of representative sampling crucial, unless a further source of uncertainty and bias is introduced.

In order to detect all cocaine metabolites, including the highly polar ecgonine methyl ester (EME), Quintela et al. developed and validated a hydrophilic interaction liquid chromatography (HILIC) method to be coupled to tandem mass spectrometry (Quintela et al., 2010). With as little as $10 \mathrm{mg}$ of specimen, experimental LODs were better than or equal to $1 \mathrm{pg} / \mathrm{mg}$. The recognition of ultra-trace amounts, at low pg/mg of hair, appears to be crucial for EME detection, because this metabolite has a very low incorporation rate into the hair shaft (Quintela et al., 2010). Recently, Thibert et al. adopted a clean-up process for hair extracts based on molecularly imprinted polymers (MIP) selective for cocaine and BZE, followed by LC-MS/MS analysis (Thibert et al., 2012). An LOD lower than $70 \mathrm{pg} / \mathrm{mg}$ was reached for both molecules, slightly above the suggested cut-off value for BZE (Cooper et al., 2012).

A fast, but only qualitative, screening method to detect cocaine and its metabolites from hair samples used MALDI-TOF technology (Vogliardi et al., 2009). The whole hair sample preparation for MALDI analysis turned out to be significantly more rapid and simpler than for GC/MS, and data acquisition in MALDI analysis is, in turn, much quicker than in GC/MS. Thus, the MALDI technique shows clear advantages over the traditional approach, when fast screening of a large number of sample is required, as the same authors subsequently demonstrated by validating and testing the new method on 304 real hair samples (Vogliardi et al., 2010). High throughput and fitfor-purpose principles both find neat application in this well-designed example of fast cocaine screening in hair samples. 


\section{B. Non-Targeted Drug Screening}

203

204

Most of the methods cited in the preceding chapter are specific for a class of drugs. The highlyfocused objective of these analytical investigations allows one to optimize sample treatment, cleanup, and chromatographic conditions in order to maximize recoveries and to meet the acceptance criteria of sensitivity and specificity that lead to accurate quantification for confirmation. However, their applicability in general drug screening for acute intoxication and autopsic analysis appears limited. In practice, these class-specific procedures find useful application when the target analytes can be predicted in advance; for example, in heroin overdose fatalities.

In the daily activity of forensic laboratories, it is quite frequent that the target analytes cannot be foreseen, so that a wide range or "general" screening of abused drugs is commonly required, as it occurs in most acute intoxications and post-mortem investigations. Whenever a multitude of candidate substances might represent the cause of intoxication or death, several analytical procedures are likely to be used on the collected biological specimen, with a direct impact on time, costs, and efficiency. For this reason, comprehensive screening procedures of multiclass drugs are progressively introduced into the analytical practice, even if the method conditions should compromise on absolute performances, in terms of recovery, sensitivity, selectivity, and accuracy, due to the presence of target analytes with widely different physico-chemical properties.

Even though hair is not the preferred biological matrix to ascertain acute intoxication, hair analysis is frequently commissioned to complete the circumstantial evidences; i.e., to ascertain whether the victim formerly abused drugs or whether any drug was administered to him/her earlier. To meet the high demand for drug screening in hair samples, toxicology laboratories are forced to update their procedures in order to target an ever-increasing number of drugs, but also to achieve rapid, simple, and sensitive testing with reduced sample preparation and fast instrumental processing so as to increase the overall sample throughput.

\section{Methods based on GC separation}

Cordero and Paterson developed and validated a GC/MS protocol to simultaneously quantify fourteen compounds, including some amphetamines, opiates, cocaine and metabolites, diazepam, and metabolite (Cordero \& Paterson, 2007). A two-step derivatization with $N$-methyl-bis trifluoroacetamide (MBTFA) and $N$-methyl- $N$-trimethylsilyltrifluoroacetamide (MSTFA) $+1 \%$ trimethylchlorosilane (TMCS) was used. Due to the general availability of GC/MS instrumentation in toxicology laboratories and the good analytical performances obtained in this study, this method 
might find useful application in a variety of clinical and forensic investigations. An interesting evolution of the preceding method was developed by Aleksa et al., that used an HS-SPME-GC/MS to improve the protocol sensitivity (Aleksa et al., 2012). Seventeen drugs of abuse, including opiates, cocaine, amphetamines, and opioids, were reacted with the same derivatizing agents described above, and were detected at LOD levels of 130-200 pg/mg. The method was validated with only 5-10 mg of hair, and was especially useful for newborn, when sample availability is limited. Among the wide screening protocols to detect common panels of abused drugs, only few comprehensive methods include THC, regardless of the instrumental technique used. Merola et al. developed a new procedure with HS-SPME and GC/MS to provide concomitant determination of several drugs of abuse, including THC, but not opiates (Merola et al., 2010). A different approach and derivatizing agent, namely heptafluorobutyric anhydride (HFBA), was used (Wu et al., 2008a) to develop a GC/MS method for the simultaneous determination of amphetamines, opiates, ketamine, and their metabolites at LODs of 30-80 pg/mg. The same group (Wu et al., 2008b) improved selectivity and sensitivity to reach the low $\mathrm{pg} / \mathrm{mg}$ level with electron capture negative chemical ionization (NCI) to detect the same derivatives.

TOF mass analyzers have also been used for broad drug screening in hair samples that take advantage of their full-scan and high-resolution capabilities. In an original study, Guthery et al. developed a GC $\times$ GC-TOF-MS procedure to provide a comprehensive qualitative drug screening of hair samples (Guthery et al., 2010). The hair extracts were subjected to derivatization with Nmethyl-N-(tert-butyldimethyl)trifluoroacetamide (MTBSTFA), which efficiently reacted with a broad range of multiclass analytes, including opiates, opioids, cocainics, and benzodiazepines. The 2D chromatographic plot obtained from $\mathrm{GC} \times \mathrm{GC}$ separation offers an extremely clear depiction of the drug content profile, as Figure 2 demonstrates (Guthery et al., 2010).

\section{Methods based on LC separation}

Although the latter GC/MS methods proved suitable to test simultaneously several classes of drugs of abuse, the need to further expand the assortment of target substances within the same protocol, without the necessity of derivatization, made the highly flexible LC-MS/MS approach to multiclass screening more profitable and more widely used than GC/MS. An LC-APCI-MS/MS method was developed, validated, and applied for the retrospective multi-parameter evaluation and distribution of eleven drugs of abuse in hair samples (Klis et al., 2007). Subsequently, ESI was more extensively utilized than APCI, as in a validated LC-MS/MS method (Miller et al., 2008) that was aimed at the simultaneous quantification of amphetamines, opiates, cocaine and metabolites, 
and diazepam and metabolites (17 compounds) in post-mortem hair samples. The use of an ion trap mass spectrometer was suggested (Bucelli et al., 2009) to screen 16 drugs of abuse in human hair. Despite the flexibility of ion traps to provide an easy switch between full-scan and MS/MS or MS modes of data acquisition, the method was not expected to provide accurate quantitative determinations, and needs further validation and a more extended panel before it might find widespread application in forensic laboratories. Di Corcia et al. proposed a simple sample extraction and direct injection into a UHPLC-MS/MS system to avoid solid-phase and liquid-liquid extraction and to achieve fast and simultaneous detection of the most common drugs of abuse, including THC, in hair samples (Di Corcia et al., 2012). Lendoiro et al. developed and validated an LC-MS/MS multiclass screening method for the simultaneous detection of 35 substances, including THC, and the other most common drugs of abuse (opiates, amphetamines, cocaine, LSD, ketamine and scopolamine) and pharmaceuticals (benzodiazepines, antidepressants and hypnotics) (Lendoiro et al., 2012). For the entire range of investigated molecules, LODs were $0.2-50 \mathrm{pg} / \mathrm{mg}$ and achieved better cut-offs than Society of Hair Testing (SOHT) recommendations. In the screening mode of operation, only one SRM transition per compound was used, but the same procedure could be transformed into a confirmation method with addition of further target-specific SRM transitions (Lendoiro et al., 2012). Confirmatory analysis of ketamine and norketamine was the objective of a recent study that used molecularly imprinted solid-phase microextraction (MISPE) followed by LCMS/MS (Harun et al., 2010).

An effective method to check for the presence of a wide set of drugs in hair samples was proposed (Nielsen et al., 2010), to validate an analytical method for the simultaneous screening and quantification of 52 common drugs and pharmaceuticals in hair with the novel UHPLC-TOF-MS technology. The panel of compounds was very broad because it included common drugs of abuse, benzodiazepines, analgesics, antidepressants and antipsychotics. The authors underlined, however, that the TOF instrument was less useful than a triple quadrupole on low-mass molecules, such as amphetamines, due to the presence of high background noise and the consequent lack of sensitivity and linearity (Nielsen et al., 2010). Similar broad drug screening, based on accurate mass measurements with LC-TOF-MS, was also achieved (Pelander et al., 2008). Also in this study, the potential to conduct a comprehensive screen (35 targeted analytes) was clearly demonstrated; however, the authors recommended more-specific instrumentation for target confirmation analyses. More recently, an automated screening method for the simultaneous identification and quantitation of 30 compounds (including most drugs of abuse, but also a few anabolic steroids, $\beta$-agonists and other pharmaceuticals) in hair samples was proposed with fast LC-TOF-MS (Domínguez-Romero et al., 2011). Progressive instrumental upgrading and method refinement provided improved 
sensitivity with TOF mass spectrometers to allow the detection of the target compounds in the low $\mathrm{pg} / \mathrm{mg}$ range (LODs ranged from $5 \mathrm{pg} / \mathrm{mg}$ to $50 \mathrm{pg} / \mathrm{mg}$ ).

General screening of unknown drugs in hair samples was performed with LC combined with a quadrupole time-of-flight mass spectrometer (LC-QTOF-MS) (Broecker et al., 2012). Systematic collection of CID (collision-induced dissociation) spectra from quadrupole-selected protonated molecule used a data-dependent acquisition mode to create a wide dataset to allow the retrospective investigation of potentially toxic substances present in the hair sample. These CID spectra were compared with an accurate-mass CID spectral library created by the authors that contained more than 2500 toxicologically relevant substances (Broecker et al., 2011; Niessen, 2011). For 24 tested illegal drugs and benzodiazepines, significantly low LODs (3-15 pg/mg) were observed. The method was applied to hair samples from 90 death cases to reveal 212 substances altogether, even if only partial agreement with the positive identifications from urine and blood analysis was found, with many unexpected drugs detected and many reported drugs not detected in hair (Broecker et al., 2012). A similar approach was also applied to hair samples (Liu et al., 2010) with an in-house spectral library of about 800 substances for the identification of unknown drugs. In this case, the hybrid $\mathrm{QqQ}_{\mathrm{LIT}}$ mass spectrometer produced low-resolution CID spectra from mass-selected precursor ions, with good sensitivity provided by the ion-accumulation capability of ion trap.

\section{Other methods}

Besides GC and LC, capillary electrophoretic methods were also occasionally used to separate drugs extracted from hair samples and to introduce them into the mass spectrometer. Capillary zone electrophoresis was combined with TOF analysis (CZE-TOF-MS) to detect four amphetamines, ephedrine, opiates, cocaine, and metabolites in hair samples. The method proved suitable for qualitative screening of all drugs below the prescribed cut-off limits, but was not fully satisfactory for accurate quantification, possibly due to the lack of deuterated internal standards (Gottardo et al., 2007; Gottardo et al., 2012). Also, another method, cation-selective exhaustive injection and sweeping micellar electrokinetic chromatography, proved compliant with the cut-off values in hair (Lin et al., 2007); however this method and the preceding study represented isolated proposals with no further application in common laboratory practice.

Quite uniquely, Jackson et al. proposed to eliminate the chromatographic separation with an ambient ionization technique, defined as low temperature plasma (LTP) ionization, to analyze biological specimens, including urine, saliva, and hair extracts (Jackson et al., 2010). The method was tested on 14 drugs that belonged to different classes (amphetamine, BZE, cannabidiol, caffeine, 
cocaine, codeine, diazepam, ephedrine hydrochloride, heroin, ketamine, methadone, 338 methamphetamine, morphine, and THC) with a linear ion trap mass spectrometer in the positive-ion 339 mode. However, LOD values were rather high $[\mathrm{ng} / \mathrm{mL}$ (i.e., $10 \mathrm{ng} / \mathrm{mL}$ ) to the $\mathrm{mg} / \mathrm{mL}$ 340 concentrations] and only one real hair sample was analyzed that showed the presence of cocaine. 341 The main focus of the study was on the potential of LTP ionization and its compatibility with 342 miniaturized and portable instrumentation for on-site screening analysis.

\section{Rape Drug Detection and Segmental Analysis}

345 Drug-facilitated crime (DFC) is a general term that involves the administration of a drug to 346 perpetrate a variety of crimes, including rape or other sexual assault, robbery, money extortion, as 347 well as the deliberate maltreatment of elderly persons or children under the influence of 348 psychotropic substances [UNODC (United Nations Office on Drug and Crime), 2011]. In most 349 DFCs, the victims are subjected to violence or nonconsensual acts while they are incapacitated by 350 the effects of a drug, often unknowingly ingested. Psychoactive substances used in DFCs might 351 alter the victim's degree of consciousness, state of awareness, judgment, and memory. Such 352 substances can make the victim vulnerable and unable to fight off the attacker, or are used to sedate 353 the victim in order to facilitate his/her transport by the perpetrator. The drugs used for these 354 criminal purposes include benzodiazepines, hypnotics, sedatives and anesthetics, drugs of abuse (such as cannabis, ecstasy, LSD, or heroin), miscellaneous drugs (e.g., scopolamine), and, most frequently, ethanol (Kintz, 2007b). The ideal substance to be dispensed to perpetrate a crime is the one that is readily available, easy to administer, able to rapidly impair consciousness, and produce anterograde amnesia (LeBeau \& Montgomery, 2010).

Among DFCs, a drug-facilitated sexual assault (DFSA) occurs when a person is subjected to unwilling sexual acts while incapacitated or unconscious, due to the effects of ethanol, drugs, and/or other intoxicating substances (generally called "rape drugs"). It is highly unlikely that the specific drug used in a DFSA could be determined only by symptoms because the depressant effects of most of these drugs are similar. This factor makes crucial identification of the administered drug in the victim's biological specimen (Negrusz \& Gaensslen, 2003). It is well-known that sexual assaults are significantly under- and late-reported. Evidently, any delay to report a DFSA implies that the victim's biological fluids (i.e., blood and urine) are not timely collected, hence rendering more difficult the toxicologists' task to identify the incapacitating agent (LeBeau \& Mozayani, 2001). Whenever the natural metabolic processes have eliminated the drug from biological fluids (typically, urine and blood), hair is the most helpful specimen to be investigated (Negrusz et al., 2000; Villain et al., 2004; Negrusz et al., 2001; Scott, 2009; Parkin \& Brailsford, 2009). 
Toxicology laboratories are frequently asked to analyze hair from victims in order to reveal allegedly administered drugs. Further information is furnished by segmental analysis to generally provide chronological setting. Head hair should be collected at least four weeks after the alleged assault [UNODC (United Nations Office on Drug and Crime), 2011]. Since the average growth rate of human scalp hair is about $1 \mathrm{~cm} /$ month, administration of a single dose can be confirmed by drug

\section{Methods based on LC-MS/MS with triple quadrupoles} detection in the corresponding segment, with no presence in the preceding and subsequent segments. The expected concentration in hair is generally in the low $\mathrm{pg} / \mathrm{mg}$ range for most drugs (Kintz, 2007b; Villain et al., 2005). Therefore, the use of highly sensitive instrumental techniques is mandatory in the investigation of DFCs (Kintz et al., 2005; Chèze et al., 2005).

The analytical strategy generally starts with a comprehensive screening for benzodiazepines and other drugs commonly involved in DFCs (Parkin \& Brailsford, 2009). In recent years, several methods have been proposed for the simultaneous analysis of a large series of pharmaceuticals and drugs with LC-MS/MS with triple-quadrupole instruments. Hegstad et al. developed a multiclass LC-MS/MS method to screen several drugs of abuse on $20 \mathrm{mg}$ of hair, which included 7aminonitrazepam, 7-aminoclonazepam, 7-aminoflunitrazepam, oxazepam, diazepam, alprazolam, and two benzodiazepines-like z-drugs (zopiclone and zolpidem) (Hegstad et al., 2008). Similarly, Irving and Dickson described an LC-MS/MS procedure to detect sedatives in hair and nail samples (Irving \& Dickson, 2007). Nine benzodiazepines, three metabolites, and zopiclone and its metabolite were selected and detected at low level in $50 \mathrm{mg}$ specimen, with LODs that ranged from $0.02 \mathrm{pg} / \mathrm{mg}$ for zopiclone to $6.00 \mathrm{pg} / \mathrm{mg}$ for temazepam. This procedure was applied to the analysis of a hair sample collected 17 months after the occurrence of a DFSA: zopiclone and its metabolite were both detected in different hair segments at concentrations below $7 \mathrm{pg} / \mathrm{mg}$ (Irving and Dickson, 2007). Our group developed an LC-MS/MS method to detect 14 pharmaceuticals, including 10 benzodiazepines, ketamine, scopolamine, zopiclone, and zolpidem with LODs that ranged from 0.2 $\mathrm{pg} / \mathrm{mg}$ for ketamine and zolpidem to $4.0 \mathrm{pg} / \mathrm{mg}$ for flunitrazepam (Salomone et al., 2012a). The method was applied to a real case of segmental analysis of the hair from two women, and showed conclusive discrimination between occasional and therapeutic administration (Salomone et al., 2012a). An interesting study of high-resolution segmental analysis on single hairs was developed to investigate the history of a multiple poisoning case with clozapine (Thieme \& Sachs, 2012). From 1-2.5 mm segments of a single hair, a highly-specific LC-MS/MS procedure allowed to detect as little as $30 \mathrm{fg}(1 \mathrm{pg} / \mathrm{mg})$ of clozapine and its metabolite norclozapine. The collection of hair samples 
after a 165 days interval, combined with the subsequent comparison of drug concentration profiles, provided an accurate estimation of hair growth rate for the selected individuals (Thieme \& Sachs, 2012).

Another LC-MS/MS method was proposed and validated (Xiang et al., 2011) for the quantitative determination of 18 benzodiazepines in $20 \mathrm{mg}$ of hair. LODs ranged from $0.2 \mathrm{pg} / \mathrm{mg}$ for estazolam to $5 \mathrm{pg} / \mathrm{mg}$ for 7-aminoclonazepam, 7-aminonitrazepam, $\alpha$-hydroxytriazolam, and $\alpha$ hydroxymidazolam. This method determined the distribution of estazolam after single dose intake in hair segments of healthy volunteers: estazolam concentrations ranged between $0.56 \mathrm{pg} / \mathrm{mg}$ and $2.60 \mathrm{pg} / \mathrm{mg}$. Two real cases were studied with the same method, where the victims of DFCs were exposed to clonazepam: the presence of this drug was found in several hair segments from victim \#1, and ranged from $1.63 \mathrm{pg} / \mathrm{mg}$ to $15.47 \mathrm{pg} / \mathrm{mg}$, and in two segments from victim \#2 at $1.31 \mathrm{pg} / \mathrm{mg}$ and $11.93 \mathrm{pg} / \mathrm{mg}$ concentrations. The metabolite 7-aminoclonazepam was found in hair segments at considerably higher concentrations than the parent drug to demonstrate the opportunity to include also the main metabolites in the benzodiazepine-screening procedure (Xiang et al., 2011). Similarly, Kim et al. established and validated an analytical method to simultaneously detect 27 benzodiazepines and metabolites plus zolpidem in hair with LC-MS/MS (Kim et al., 2011). The absolute LODs ranged from $0.005 \mathrm{ng}$ (zolpidem) to $0.5 \mathrm{ng}$ (bromazepam and chlordiazepoxide). The protocol was successfully applied to five forensic cases of either DFCs or benzodiazepines abuse, together with a study on drug incorporation into the hair of rats. As model for human hair, it was proved that rat-hair pigmentation does not have any significant effect on the degree of benzodiazepines (and metabolites) deposition in hair. As a matter of fact, the incorporation rates of other classes of drugs proved to depend on the hair pigmentation, to create ethnic discrimination (Gambelunghe et al., 2007; Polettini et al. 2012). The panel of screened compounds was expanded further by Lendoiro et al., who recently presented a target multi-analyte LC-ESI-MS/MS screening method to detect 35 licit and illicit drugs, including THC, in $50 \mathrm{mg}$ hair samples (Lendoiro et al., 2012). Among the screened substances, zolpidem, zopiclone, and 12 benzodiazepines were detected at LODs that ranged from $2 \mathrm{pg} / \mathrm{mg}$ (7-aminoflunitrazepam, flunitrazepam, lorazepam, lormetazepam, nordiazepam, and diazepam) to $10 \mathrm{pg} / \mathrm{mg}$ (oxazepam, clonazepam, and tetrazepam). Analysis of seventeen hair specimens from various forensic cases demonstrated the method applicability: several illicit and prescription drugs were tentatively identified, and subsequently confirmed with two SRM transitions in place of one (Lendoiro et al., 2012). In all the cited LCMS/MS procedures, triple-quadrupole instruments were used, and proved the efficiency of this instrument for wide multi-analyte screening. Similar LC-MS/MS configuration was also used in confirmation methods specifically designed to determine acepromazine (Gaulier et al., 2008), 
diazepam, nordiazepam and tetrazepam (Laloup et al., 2007), sildenafil (Kintz et al., 2009), and gamma-hydroxybutyrate (Stout et al., 2010) in hair samples, with the purpose to investigate DFSA cases.

\section{High-resolution mass spectrometers}

Mass spectrometers other than triple quadrupoles were used for wide-range screening and confirmation analysis. Mass analyzers with high-resolution capability and accurate mass detection, such as TOF-MS and FTICR-MS, are particularly suited for a-posteriori reevaluation of the presence of drugs not considered in a preliminary target analysis. UHPLC-TOF-MS and UHPLCMS/MS were both used in the development of a specifically validated confirmation protocol, aimed to quantification of triazolam in an emblematic case of DFC (Stybe Johansen and Dahl-Sørensen, 2012). In the hair segments $(2 \mathrm{~cm})$ that corresponded to the period of the alleged assaults, the presence of triazolam was revealed at a concentration of $1.0 \mathrm{pg} / \mathrm{mg}$. A wider range of analytes was covered in a high-resolution mass spectrometric (HRMS) drug screening with TOF-MS (Pelander et al., 2008). The authors initially developed an analytical strategy for the comprehensive screening of drugs and doping agents in urine, but afterward the applicability of the method to other matrices, such as keratin, was also studied. Although the potential of the LC-TOF-MS approach to obtain a comprehensive drug screening in the hair of drug addicts was clearly proved, the study did not clarify whether the method sensitivity was high enough to detect drug traces from single intake. A UHPLC-TOF-MS screening procedure previously cited (Nielsen et al., 2010) targeted 52 analytes, among which 13 benzodiazepines and metabolites plus zaleplon, zolpidem, and zopiclone. For the screened molecules, homogeneous LODs were obtained that ranged from $10 \mathrm{pg} / \mathrm{mg}$ for diazepam and zolpidem, to $40 \mathrm{pg} / \mathrm{mg}$ for 7-aminoflunitrazepam and lorazepam. The method was applied to 15 autopsy hair samples. In most strands, several benzodiazepines were identified. Although accuratemass analysis provided comparable selectivity to multiple SRM transitions from a triple-quadrupole instrument, the authors recommended positive findings to be confirmed with an additional method (Nielsen et al., 2010).

Orbitrap technology was also used for HRMS multi-target screening of hair samples (Vogliardi et al., 2011). The purpose of this study was to develop and validate a specific method based on LCHRMS for the simultaneous detection in $50 \mathrm{mg}$ hair strands of 28 benzodiazepines (and metabolites) registered in the Italian market. The "z-drugs" were not included, despite the fact that zolpidem is significantly prescribed among the Italian population. The use of a hybrid linear ion trap-Orbitrap mass spectrometer yielded LODs from $0.5 \mathrm{pg} / \mathrm{mg}$ (clotiazepam) to $10 \mathrm{pg} / \mathrm{mg}$ 
(delorazepam). The validated procedure was applied to hair samples from suspected benzodiazepine consumers and showed its suitability for screening and identification of the target compounds. Indeed, the potential of such an instrument includes full-scan exact-mass measurement of protonated molecule ions and CID fragment ions, isotopic pattern recognition, and ion ratio determination between molecular and fragment ions. Recently, the same group developed and validated an analogous method to detect 28 substances that belonged to several drug classes: cocaine, amphetamines, opioids, benzodiazepines, antidepressant, and hallucinogens. Although a mass spectrometric approach similar to the one previously described was applied, a new and faster extraction process on micropulverized hair allowed reliable identification and quantification of the target drugs on as little as $2.5 \mathrm{mg}$ of hair samples (Favretto et al., 2012). The combined use of fullscan HRMS and CID offered the possibility of retrospective analysis to detect previously untargeted substances.

\section{Biomarkers for Alcohol Abuse}

An important goal of forensic toxicology and clinical medicine is to identify appropriate biological markers of ethanol consumption to evaluate harmful drinking and to ascertain alcohol abstinence (Pragst \& Balikova, 2006). The determination of direct ethanol metabolites, such as ethyl glucuronide (EtG), ethyl sulfate (EtS), fatty acid ethyl esters (FAEEs), and phosphatidylethanol (PEth), in different biological matrices currently represents the most-valid strategy to provide unbiased evidence of chronic alcohol abuse. Among direct biomarkers, FAEEs and EtG can be detected in a variety of matrices, including hair (Pragst \& Balikova, 2006), and allow one to gain information on alcohol consumption for several months. Hair analysis proved very useful to monitor ethanol use/abstinence, workplace testing, driving license reissue/renewal, child custody, divorce proceeding, withdrawal treatment (Politi et al., 2006), and post-mortem or pre-natal alcohol exposure investigation (Bendroth et al., 2008; Pragst \& Yegles, 2008), even if the straightforward interpretation of analytical results has been fiercely questioned and warning about possible intra- and inter-individual variability has been raised (Tagliaro et al., 2011).

According to the Society of Hair Testing, analysis of the proximal $3 \mathrm{~cm}$ hair segment is recommended in order to avoid partial loss of the analytes. The suggested cut-off values that support a diagnosis of chronic excessive alcohol consumption are $30 \mathrm{pg}$ EtG/mg scalp hair, and 0.5 ng FAEEs/mg scalp hair, respectively; both measured in the $0-3 \mathrm{~cm}$ proximal segment (Kintz, 2012b). Figure 3 shows the effectiveness of the $30 \mathrm{pg}$ EtG/mg cut-off value (horizontal line) to discriminate harmful drinkers (red dots) from low-risk drinkers and non-drinkers (yellow and green dots), whereas the cut-off value (vertical line) commonly adopted for an esteemed indirect 
biomarker, such as carbohydrate-deficient transferrin (CDT), proved to have reasonable specificity but largely insufficient sensitivity, that led to about 50\% false negative results (Pirro et al., 2011a).

In contrast to other forensic applications, a very small number of direct alcohol biomarkers represent for toxicologists the target analytes in hair samples, essentially FAEEs and EtG. Consequently, considerable effort has been devoted to their extraction procedures and instrumental arrangements, in order to obtain continuously improving analytical performances and, above all, minimal LOD and LOQ values. In particular, the refinement of existing methods was addressed to (i) increase the laboratory throughput, with a reduction of sample preparation steps, analysis-time, and costs, (ii) increase the sensitivity and detection capability, so as to accurately quantify minute biomarkers hair concentrations (in the $\mathrm{pg} / \mathrm{mg}$ range), especially to ascertain alcohol abstinence, (iii) improve the method validation protocols in order to consider all potential sources of bias and uncertainty and assure highly reliable analytical results. Under such circumstances, quite traditional instrumentations, such as triple-quadrupole mass spectrometers in the SRM mode, combined with either GC or HPLC, were almost uniquely utilized, because they assure sensitive and accurate quantification of few pre-determined target analytes.

\section{FAEE determination in hair samples}

Due to the relatively high volatility and hydrophobicity of FAEEs, HS-SPME-GC/MS is the suggested method-of-choice for the routine determination of ethyl myristate (E14), ethyl palmitate (E16), ethyl stearate (E18), and ethyl oleate (E18:1) in hair extracts (Kintz, 2012b). Among over 20 ethyl esters detected in hair, these four selected analytes were found to best reflect harmful drinking (Pragst et al., 2001). In brief, deuterated internal standards are added to decontaminated hair samples, and subsequently extracted overnight with a heptane-dimethylsulfoxide mixture. The hydrocarbon layer is evaporated and the residue dissolved into a phosphate buffer, on which HSSPME is executed. The SPME fiber is analyzed with GC/MS in the electron impact (EI) ionization mode under SIM conditions.

Over time, laboratories interested in testing for FAEEs adopted the protocol (Pragst et al., 2001), with small changes for in-house revalidation of the method and to optimize the experimental conditions, to determine E14, E16, E18 and E18:1, with the purpose to better clarify the reliability, limitations, and potential of FAEE biomarkers in hair to ascertain harmful drinking. Later, Pragst and Yegles presented an updated procedure, with detection limits ranging between 0.003-0.01 ng/mg, as determined on $30 \mathrm{mg}$ of hair (Pragst \& Yegles, 2008). 
Kulaga et al. investigated the potential effect of hair pigmentation on FAEEs incorporation into the keratin matrix, and used, for the first time, hair collected from rats as a model matrix for FAEE production and hair inclusion (Kulaga et al., 2009). As expected for neutral and lipophilic molecules such as FAEEs, they concluded that FAEE concentration in hair does not depend on hair pigment. However, they noted that FAEE hair concentration in rats was much lower than that typically observed in humans, at comparable dosage, but still measurable with the analytical protocol adopted. Gareri et al. used the same analytical method to study the potential bias from use of ethanol-containing hair-care products on FAEE concentration measured on hair of a noncompliant female population (Gareri et al., 2011). From the comparison of FAEE and EtG concentrations in the same hair samples, they concluded that use of hair-care products that contain ethanol (even at low percentage; i.e., less than $10 \%$ by volume) might affect FAEE levels in real hair samples, with experimental values from approximately 0.5 to $5.0 \mathrm{ng} / \mathrm{mg}$, to possibly produce false non-compliant outcomes (Gareri et al., 2011). Süsse et al. considered the experimental results from as many as 1872 hair samples from forensic cases (Süsse et al., 2012). They deduced that (i) the use of hair spray might affect (increase) detected FAEEs, but not EtG, hair concentrations; (ii) conversely, bleaching and dying hair treatments might affect (decrease) EtG, but not FAEEs, hair concentrations, whereas (iii) hair gel, hair wax, oil, and grease have apparently no influence on FAEEs and EtG.

Süsse et al. reported a method with detection limits ranging from 0.008 to $0.026 \mathrm{ng} / \mathrm{mg}$, as measured on 20-50 mg hair samples (Süsse et al., 2010). These analytical features allowed the same authors to accurately measure the basic levels of hair FAEEs in strict teetotalers: the results showed that $20 \%$ of abstainers $(\mathrm{N}=242)$ presented total a FAEE concentration that exceeded the cut-off values of 0.5 or $1.0 \mathrm{ng} / \mathrm{mg}$, as a function of the examined hair length (Süsse et al., 2012). In contrast, Albermann et al. reported total a FAEEs concentration lower than $0.2 \mathrm{ng} / \mathrm{mg}$ for $74 \%$ of drivers who self-claim as abstinent $(\mathrm{N}=160)$, whereas only $9 \%$ of these samples showed a FAEE concentration that exceeded $0.5 \mathrm{ng} / \mathrm{mg}$, among which some true-positive cases (Albermann et al., 2011).

Lately, Hastedt et al. updated and revalidated the HS-SPME-GC/MS method originally developed by Pragst et al. (Hastedt et al., 2012; Pragst et al., 2001). Better detection limits were achieved (0.004-0.014 ng/mg), although an expanded time to complete the chromatography cycle was needed (over $30 \mathrm{~min}$ ) than in the original method.

On the other hand, Caprara et al. and Kulaga et al. described a different analytical strategy to quantify a larger number of FAEEs in neonatal human and animal hair samples, with ethyl heptadecanoate as the internal standard (Caprara et al., 2006; Kulaga et al., 2006). They adopted 
solid-phase extraction (SPE) in place of SPME to isolate FAEEs from hair extracts, followed with either GC/MS or GC/MS/MS in the chemical ionization (CI) mode, with isobutene as the ionizing gas. Unlike EI, which typically leads to an extensive, but nearly identical, fragmentation patterns for all FAEEs, CI virtually generates only the protonated molecule ion, which is distinctive for each ethyl ester. MS/MS performed in an ion-trap device clearly adds specificity to the method. In choosing CI rather than EI for the analysis, good detection limits were achieved $(<2.4-6.6 \mathrm{pg} / \mathrm{mg})$, only recently outperformed, as reported above. FAEE-analysis profile was also expanded to six, rather than four esters, by adding ethyl laureate (E12), and ethyl palmitoleate (E16:1), and cumulative analysis of all commonly occurring FAEEs in biological matrices was suggested to provide a more effective way to identify neonates with suspected pre-natal exposure to ethanol (Caprara et al., 2006; Kulaga et al., 2006).

By taking advantage of the preceding experiences, Zimmermann and Jackson proposed a globally revisited analytical method to determine five FAEEs (E12, E14, E16, E18, and E18:1) in human hair, and aimed to combine high sample throughput with analytical performances (Zimmermann \& Jackson, 2010). Instead of extensive washing procedures and an overnight extraction, followed by a 20 or 40 min-long GC/MS analysis, they proposed a method that required a total analysis-time of less than 1 hour, including a 15-minute sonication-extraction procedure, followed with HS-SPME-GC/MS/MS, performed with a quadrupole ion trap. Positive-CI combined with MS/MS provided high selectivity and detection capability, despite a total chromatography cycle time of only $9 \mathrm{~min}$. The limits of detection ranged from 0.002 to $0.030 \mathrm{ng} / \mathrm{mg}$ (Zimmermann \& Jackson, 2010). More recently, Politi et al. developed a sensitive (LOQ $=0.01 \mathrm{ng} / \mathrm{mg}$ for each FAEE) analytical protocol for FAEE determination in hair, based on the widespread SPE and GCEI-MS techniques (Politi et al., 2011). These techniques are affordable by any toxicology laboratory - a key feature to make this determination routine. Four ethyl esters (E14, E16, E18, and E18:1) were chromatographically separated in about $16 \mathrm{~min}$. Faster chromatographic analysis is prevented by the need to obtain well-separated peaks and no interferences, because FAEEs share most EI fragment ion signals.

\section{EtG determination in hair samples}

EtG is a polar substance with a relatively low molecular weight. Due to these attributes, a singlestage of MS analysis is generally insufficient to comply with the low detection limits in the pg/mg range required for its determination in a complex keratinic matrix. Either GC/MS/MS after derivatization or LC-MS/MS are the methods of choice for EtG determination in hair samples; both 
are capable to minimize the background interferences and to achieve the optimal detection capability and high specificity, required in toxicological and forensic applications. The limitations of the simple GC-EI-MS approach for EtG determination in hair samples is indirectly demonstrated by a recently developed method, which barely reaches an LOD of $100 \mathrm{pg} / \mathrm{mg}$ with as much as 100 mg of hair in the SIM acquisition mode (Álvarez et al., 2009). This LOD value is considerably higher than the established cut-off limit to evidence alcohol use disorders (Kintz, 2012b).

Although the simplicity and progressively increasing performances of ESI make the LC-MS/MS approach prevalent nowadays, several highly-efficient GC/MS/MS methods have been recently developed and updated to fulfill the legal requisites entailed by regulations of many countries. Paul et al. proposed and validated a GC/MS/MS procedure, with EI, triple-quadrupole mass spectrometer, and SRM (Paul et al., 2008). The instrumental analysis followed water extraction, SPE purification, and derivatization with N,O-bis[trimethylsilyl]trifluoroacetamide (BSTFA). An LOD of $5 \mathrm{pg} / \mathrm{mg}$ was obtained, and the chromatography cycle was $7.30 \mathrm{~min}$ (Paul et al., 2008). A modified protocol was reported by the same group in 2011, in which the SRM transitions for GHB were added to the same analytical protocol, so as to detect EtG and GHB within a single chromatographic procedure. This combination is of importance in the investigation of drugfacilitated crimes, because GHB and alcohol are frequently combined substances used to perpetrate these offences (Paul et al., 2011).

Large-volume injection (LVI) GC-EI-MS/MS was proposed to increase the sensitivity in hair EtG determination. The authors suggested that, upon optimization of streaming volume, purge time, programmable temperature vaporization (PTV) temperature, injection volume, and other experimental parameters, the detection capability can be improved by at least 1-2 orders of magnitude, as compared to the traditional approach (Shi et al., 2010). Even so, they estimated an LOD value of $5 \mathrm{pg} / \mathrm{mg}$, with $20 \mathrm{mg}$ of hair, as in the method previously cited (Paul et al., 2008).

NCI with methane was proposed as an alternative to EI within a GC/MS/MS protocol in order to improve analytical sensitivity (Kharbouche et al., 2009). A high cross-section for electron capture was induced by double derivatization of $\mathrm{EtG}$ with perfluoropentanoic anhydride. The choice of two selective SRM transitions allowed high specificity in the analyte identification, despite the fast GC protocol. The estimated LOD value for EtG was $3 \mathrm{pg} / \mathrm{mg}$, with $30 \mathrm{mg}$ hair. This analytical protocol was adopted to analyze rat hair samples, so as to evaluate any potential hair-pigmentation effect on EtG incorporation into the keratin matrix. No statistically significant differences were observed between pigmented and non-pigmented hair (Kharbouche et al., 2010). The same group also evaluated the possible effect of hair-care products on EtG incorporation (Sporkert et al., 2012). 
A similar GC-NCI-MS/MS approach was also proposed (Agius et al., 2010), but the final EtG enrichment was performed with HS-SPME, after derivatization with heptafluorobutyric anhydride. HS-SPME injection yielded further clean-up in the headspace extraction and accumulation of the derivatized analyte on the fiber, with a significant decrease of the LOD value: $0.6 \mathrm{pg} / \mathrm{mg}$, with 10 mg hair (Agius et al., 2010). No further improvements were recorded with nonafluorobutyrric anhydride for EtG derivatization instead of heptafluorobutyric anhydride. A similar LOD value (0.7 $\mathrm{pg} / \mathrm{mg}$ ) was also achieved (Kerekes et al., 2009), from 20-30 mg of hair, although NCI was followed by single MS detection, with two monitored ions in the SIM acquisition mode. The EtG concentration was in hair collected from several parts of the body for the same subjects determined and compared (Kerekes et al., 2009).

Even though the GC/MS/MS procedures for EtG determination in hair proved to be accurate and highly sensitive, especially when electron capture was used, most toxicology laboratories currently prefer LC-MS/MS, because the sample-purification steps are easier and no derivatization is needed. These simplifications substantially contribute to reduce the work and time for sample processing - a crucial aspect in laboratories with high sample load.

Although the first LC-MS/MS method dates back to 2002, the real bloom of analytical procedures based on LC separation and ESI started in 2007. ESI is commonly used in the negative ion mode because of the acidic nature of EtG. Among the large variety of proposed methods, different sample-preparation steps, amounts of analyzed hair, and chromatographic conditions were adopted that yielded excellent selectivity and LOD values between 1 and $10 \mathrm{pg} / \mathrm{mg}$. The proposed methods proved highly reliable to support evaluation of harmful drinking (Morini et al., 2009; Marques et al., 2010; Pirro et al., 2011a; Agius et al., 2012); monitor total abstinence (Kronstrand et al., 2012); study hair-treatment effects that possibly lead to partial disappearance of EtG from the keratin matrix (Morini et al., 2010); and evaluate the effect of individual and external parameters on EtG accumulation and persistence in hair, so as to define the boundaries and to exclude conditions for its use as a biomarker of ethanol consumption (Pragst et al., 2010, Süsse et al., 2012; Hastedt et al., 2012; Cabarcos et al., 2012; Pirro et al., 2011b). Almost all these methods made use of triplequadrupole instruments to monitor SRM transitions between the deprotonated molecule ion of EtG $(\mathrm{m} / \mathrm{z} 221)$ and two fragment ions $(\mathrm{m} / \mathrm{z}, 75,85)$. For EtG determination in hair samples, a detailed list of MS conditions used in the cited articles is presented in Table 1. Conditions for GC and LC methods are reported.

The major critical point of hair EtG determination with LC-ESI-MS/MS methods is the possible occurrence of matrix effects (Matuszewski et al., 2003; Peters \& Remane, 2012), especially when a non-polar stationary phase is used for LC separation; i.e., EtG eluted quickly. In any case, matrix 
effects need to be carefully evaluated during method development and validation (Lamoureux et al., 2009; Tarcumnicu et al., 2010; Cabarcos et al., 2012). Several authors proposed to modify previously developed analytical protocols in order to improve sample clean-up (essentially with SPE) and consequently reduce ion-suppression and increase the signal-to-noise ratio. Politi et al. reported an extreme case, in which SPE clean-up proved useful to decrease ion suppression and identify EtG traces in three hair segments of a known alcoholic, 27 years after his death (Politi et al., 2008).

Carbon-blend SPE columns, based on porous graphitic material, are suitable for the extraction of EtG from complex matrices such as hair (Lamoureux et al., 2009). Besides, the use of silica HPLC columns, in place of reversed-phase columns, allows one to elute EtG with a mobile phase that contain a high percentage of organic solvent and low percentage of water, to improve ESI efficiency (Lamoureux et al., 2009). An alternative strategy, proposed previously, made use of postcolumn addition of acetonitrile to the eluate. For the same goal, the use of HILIC coupled to mass spectrometry was proposed and successfully applied for the analysis of EtG in hair (Kintz et al., 2008; Tarcumnicu et al., 2010). Recently, Albermann et al. proposed a modification of their method for EtG determination in hair, aimed to solve occasional chromatographic problems during routine work (Albermann et al., 2012). The new method used an LC column with stationary phase composed of $100 \%$ porous graphitic carbon, instead of the reversed-phase LC column previously used (Albermann et al., 2010). However, the new method led to a longer analysis-time and slightly higher LOD value $(1.7 \mathrm{pg} / \mathrm{mg})$ than previously reported $(1 \mathrm{pg} / \mathrm{mg})$.

\section{E. Antidoping Analysis}

In amateur and professional sport, the artificial enhancement of the athletic performance with drugs or forbidden practices is subjected to systematic control. Doping controls in sport events are managed by the World Anti-Doping Agency (WADA), through tests of urine or blood sample collected from athletes in and out of competition. Hair analysis is currently not accepted as an alternative to urine and blood analysis for doping control (WADA, 2012) for several reasons, including its heterogeneous nature, the uncertainty on mechanisms that regulate drug incorporation, and alleged diversity in occurrence and extent of these mechanisms among different ethnic groups (Kintz, 2007a). However, it is evident that hair analysis would be of practical significance in several circumstances, because it provides complementary information with respect to urine and blood testing (Gaillard et al., 2000; Kintz, 2003). In fact, urine and blood analyses detect recent drug intake, but cannot distinguish between chronic use or single exposure, whereas hair analysis can offer this distinction (Kintz, 2007a). Indeed, doping agents are partially excreted in the sweat and 
incorporated into growing hair, and remain stable for long periods of time. Thus, hair testing might offer a large detection window to allow long-term detection of prohibited drugs and a retrospective estimation of the intake period. This opportunity appears to be particularly important to detect outof-competition doping treatments; for example, in the athletes who use anabolic substances during the training period and interrupt their intake long before the start of competition (Kintz et al., 2000).

The need for equal conditions for all athletes and the freedom for them to make use of whatever cosmetic treatment, including complete body shaving, prevented any official use of hair analysis in doping controls. For example, the incorporation rates of prohibited drugs was proved to depend on the hair pigmentation, to possibly lead to unequal conditions in doping control, and consequently ethnic discrimination (Gambelunghe et al., 2007). In addition, hair washing, discoloring, and tinting appear to influence the drug concentration measured in hair, as well as their distribution within the hair longitudinal axis (Kintz, 2003). Nevertheless, the potential of hair analysis for antidoping purposes is so strong that several analytical methods were developed in the past to detect doping agents in hair specimens (Kintz, 2007a).

In the last five years, a limited number of analytical methods was additionally developed, with the main objective to measure anabolic androgenic steroid (AASs) level in human hair with gas or liquid chromatography coupled to high-resolution mass spectrometry or tandem mass spectrometry (Anielski, 2008; Gambelunghe et al., 2007; Deshmukh et al., 2010). A complex challenge was addressed to exploit hair analysis to distinguish between natural production of endogenous steroids (e.g., testosterone) and exogenous uptake of the same steroids (Shen et al, 2009; Pozo et al, 2009, Deshmukh et al. 2012). AASs represent the most frequently detected class of substances in out-ofcompetition doping testing, because they are widely abused to increase strength and lean body mass, and also lead to reduced recovery periods (Thieme et al., 2000; Pozo et al., 2009; Deshmukh et al. 2010). AASs are also utilized to speed up muscle growth in cattle and horses (Gaillard et al., 1999; Anielski, 2008). AAS detection in hair specimens is more widely accepted in animals than in humans; it still receives a considerable interest in the scientific research, as demonstrated recently (Nielen et al., 2011) in which hormone and veterinary drug screening and, in general, forensic investigations can benefit from the recent developments in desorption electrospray ionization (DESI) mass spectrometry (Takáts et al., 2004). DESI allowed bovine hair analysis with very limited sample preparation: after rapid ultrasonic liquid extraction, a few microliters of supernatant were deposited onto a glass or PTFE surface, and dried, before DESI analysis. Full-scan and $\mathrm{MS}^{3}$ experiments with a linear ion trap MS were used to detect estradiol benzoate, testosterone cypionate, and testosterone decanoate to demonstrate the general feasibility of rapid screening and 
detection of anabolic steroid intact esters. Quite high levels (300-800 $\mathrm{g} / \mathrm{kg}$ ) of steroid esters are generally present in hair samples from controlled and illegally treated animals (Nielen et al., 2011).

Anielski published a method to detect anabolic steroids and their esters in hair material (Anielski, 2008). After hair extraction (sonication, methanol, $4 \mathrm{~h}, 50^{\circ} \mathrm{C}$ ) and LLE raw purification, a refined sample clean-up procedure was carried out with HPLC. The residue was subsequently derivatized, and the trialkylsilyl steroid derivatives were analyzed with GC/HRMS and GC/MS/MS to yield LOD values between 0.1 and $5.0 \mathrm{pg} / \mathrm{mg}$. The method was successfully applied to real samples to detect testosterone propionate in the hair of treated horses. Unlike a urine specimen, the parent drugs instead of their metabolites are more frequently detected in hair samples.

The goal to determine the natural occurrence of anabolic steroids in hair, and their biological variability within selected populations of individuals, led Shen et al. to develop a specific GC/MS/MS method to simultaneously identify testosterone, epitestosterone, androsterone, etiocholanolone, and dehydroepiandrosterone (DHEA) (Shen et al., 2009). After alkaline digestion $\left(\mathrm{NaOH}, 1 \mathrm{~N}, 10 \mathrm{~min}, 90^{\circ} \mathrm{C}\right)$, LLE extraction with diethylether was carried out. The extracts were derivatized with a MSTFA/iodotrimethylsilane/dithioerythritol mixture (10:5:5, v/v/w) and analyzed with GC/MS/MS/SRM. The method was fully validated and applied to 80 subjects (39 males, 30 females, 11 children). The sensitivity of the method (LODs between 0.1 and $0.2 \mathrm{ng} / \mathrm{mg}$ ) proved sufficient to determine the physiological concentration ranges for the selected endogenous steroids in the three populations, so as to address the interpretation of non-compliant results from alleged steroid abuse (Shen et al., 2009).

An analogous GC/MS/MS method was previously developed for the simultaneous determination of methyltestosterone, nandrolone, boldenone, fluoxymesterone, cocaine, and benzoylecgonine, and was applied to hair samples from seven athletes with reported AAS abuse (Gambelunghe et al., 2007). All anabolic steroids included in the procedure could be detected with a $10 \mathrm{pg} / \mathrm{mg}$ detection limit. Methyltestosterone, nandrolone, boldenone, and fluoxymesterone were detected in real samples between $12 \mathrm{pg} / \mathrm{mg}$ and $37 \mathrm{pg} / \mathrm{mg}$.

A specific LC-MS/MS method was developed for the clinical determination of testosterone undecanoate in hair samples (Pozo et al., 2009). After decontamination and incubation in a tris(2carboxyethyl)phosphine hydrochloride solution $\left(1.5 \mathrm{~h}, 50^{\circ} \mathrm{C}\right)$ within an ultrasonic bath, a double LLE with n-pentane was performed. The reconstituted residue was injected into an LC-MS/MS triple-quadrupole mass spectrometer with APCI source. The proposed fragmentation pattern of testosterone undecanoate is shown in Figure 4. The method was applied to hair samples from three patients treated with testosterone undecanoate and led to detected concentrations of $0.4,1.6$ and 8.4 $\mathrm{pg} / \mathrm{mg}$. 
The group of Naughton and coworkers recently published two sensitive LC-MS/MS methods for the detection of various steroids in human hair samples. In the first study, nandrolone and stanozolol were determined in human hair for the first time (Deshmukh et al., 2010). After alkaline digestion $\left(\mathrm{NaOH}, 1 \mathrm{M}, 15 \mathrm{~min}, 95^{\circ} \mathrm{C}\right)$ and LLE with n-pentane, the extracts were injected into a UHPLC system interfaced to a triple-quadrupole mass spectrometer with ESI source. From $20 \mathrm{mg}$ aliquots of hair, LOD values of $0.5 \mathrm{pg} / \mathrm{mg}$ for stanozolol and $3.0 \mathrm{pg} / \mathrm{mg}$ for nandrolone were obtained. The method was successfully applied to 19 hair samples previously tested positive with ELISA (enzyme-linked immunosorbent assay) screening to reveal seven false-positive results and to confirm one sample positive to nandrolone $(14.0 \mathrm{pg} / \mathrm{mg})$ and eleven samples positive to stanozolol (concentration range $5.0 \div 86.3 \mathrm{pg} / \mathrm{mg}$ ). The second study used a similar experimental approach to develop a highly sensitive LC-MS/MS method to determine testosterone and epitestosterone in hair samples (Deshmukh et al., 2012). The natural abundance ratio of these steroids $(\mathrm{T} / \mathrm{E})$ is significantly modified by any exogenous intake of testosterone. Low LOD values $(0.1 \mathrm{pg} / \mathrm{mg}$ and $0.25 \mathrm{pg} / \mathrm{mg}$ for testosterone and epitestosterone, respectively, from $50 \mathrm{mg}$ of hair) allowed the determination of physiological testosterone and epitestosterone levels on 75 real samples, which ranged from 0.70 to $11.81 \mathrm{pg} / \mathrm{mg}$ in males and 0.33 to $6.05 \mathrm{pg} / \mathrm{mg}$ in females (testosterone); from 0.63 to $8.27 \mathrm{pg} / \mathrm{mg}$ in males and 0.52 to $3.88 \mathrm{pg} / \mathrm{mg}$ in females (epitestosterone).

\section{FUTURE TRENDS OF HAIR ANALYSIS}

\section{A. New Designer Drugs}

In the last years, forensic laboratories have been challenged worldwide with the puzzle to detect a variety of new psychoactive substances. These compounds, occasionally called either designer drugs or legal highs, have little or no previous history of medicinal use. Amphetamine derivatives, with backbone molecular structures of piperazine and cathinone, initially represented the main group of designer drugs. Almost at the same time, another wide group of compounds, known as synthetic cannabinoids, appeared on the black market. A very rapid proliferation of new psychoactive substances of these and other classes followed in recent years. Although most of the latest drugs act as central nervous system (CNS) stimulants, their assorted chemical structures range from derivatives of pipradrol, ketamine, phencyclidine, arecoline, aminopropylbenzofuran, ring- 
substituted aminoindans, thiophenyl bioisosteres of methamphetamine, as well as compounds structurally related to cocaine (King \& Kicman, 2011).

Reference standards for most designer-drugs metabolites are not yet commercially available. That lack of standards poses a serious and longstanding challenge to toxicological laboratories to develop analytical procedures to detect their presence in a variety of biological matrices. Nevertheless, several analytical methods were developed to determine some of these new psychoactive substances and/or their metabolites in either blood or urine (Moran et al., 2011; Grigoryev et al., 2011; Dresen et al., 2011; Teske et al., 2010; Wintermeyer et al., 2010; Beuck et al., 2011; Kraemer et al., 2009; Sobolevsky, Prasolov, \& Rodchenkov, 2010). On the other hand, only very few studies have been published to date that describe protocols for the detection of these substances in hair samples.

In a Letter to the Editor, Torrance and Cooper reported the detection of mephedrone in hair samples at 4.2 and $4.7 \mathrm{ng} / \mathrm{mg}$ concentration with a ISO/17025 accredited method, but details on the analytical method used and comments or interpretations of results were not included (Torrance \& Cooper, 2010). A specific and accurate method for mephedrone detection with GC/MS was proposed (Martin et al., 2012). The study reported that 67 real hair samples were analyzed - 10 were found positive, with mephedrone concentrations from 0.2 to $313.2 \mathrm{ng} / \mathrm{mg} ; 8$ of them were below 6 $\mathrm{ng} / \mathrm{mg}$. As for amphetamines, it is deduced that mephedrone concentrations in hair are likely in the $\mathrm{ng} / \mathrm{mg}$, not $\mathrm{pg} / \mathrm{mg}$, at least in the cases of repeated abuse (Martin et al., 2012). Detection of piperazine-like compounds was meticulously described (Barroso et al., 2010b). This study was the first to report the development and validation of an analytical method for the determination of three phenylpiperazines in hair samples. Trimethylsilyl derivatives were determined with GC/MS, and the method was applied to autoptic samples and samples collected from subjects under psychiatric evaluation.

The first multiclass screening for synthetic cannabinoids in hair samples was published recently (Salomone et al., 2012b). In this study, a UHPLC-MS/MS procedure was developed and validated to determine the possible presence of five synthetic cannabinoids (i.e., consistently present in the black market during the period considered: JWH-018, JWH-073, JWH 200, JWH-250, and HU210) in 179 real hair samples collected from previously proven Cannabis consumers (Figure 5). The fourteen $(7.82 \%)$ samples found positive to at least one synthetic cannabinoid demonstrated significant diffusion among drug abusers. Concentrations of synthetic cannabinoids ranged from 0.50 to $730 \mathrm{pg} / \mathrm{mg}$. Although the published screening was limited to relatively few compounds, its UHPLC-MS/MS approach makes further method expansion feasible, so as to include new substances that are continuously introduced into the black market. 


\section{B. Future Trends Driven by Instrumental Developments}

843

After years of pioneering studies, hair analysis for toxicology applications represents nowadays a reliable and well-established means of forensic investigation. The Society of Hair Testing periodically meets to exchange scientific experiences, establish new protocols, and draw up consensus documents to carefully guide conclusions and legal judgments on the basis of experimental results from hair analysis.

Whereas instrumental sensitivity and overall method specificity have been for years key issues of experimental studies and analytical method developments, the latest instrumentation for sample treatment, chromatographic separation, and mass spectrometric detection substantially fulfills these requirements, and frequently provides LOD and LOQ values largely better (i.e., lower) than the expected drug concentrations in hair. Indeed, various sources of individual variability, including genetic polymorphisms, metabolic disorders, diet, use of cosmetics, and environmental exposure, are likely to represent more-important causes of bias and incorrect reporting than insufficient sensitivity and specificity. For example, the LOD for EtG in hair will soon break the $1 \mathrm{pg} / \mathrm{mg}$ limit and will open up a reflection on the possible origins of this alcohol metabolite other than ingestion of alcoholic beverages. Moreover, the chance of external contamination of hair samples and extracts grows with increasing method sensitivity, in turn becoming a major concern during the experimental method validation.

Other issues, basically linked to one another, are becoming increasingly important in forensic hair analysis. These issues are: multiresidue and multiclass potential, high throughput, and costs of analysis. Capability of simultaneous multiclass analysis is essential in broad drug screening of unknown analytes, because in most acute intoxications and post-mortem investigations the ingested substance cannot be anticipated; however, this feature also contributes to dramatically decrease the number of analysis for each sample, with a resultant cost lowering. Modern UHPLC-MS/MS instruments with triple-quadrupole analyzers can already simultaneously detect more than one hundred substances in just a few minutes, and QuEChERS-like procedures to extract hair samples are rapidly spreading within toxicology laboratories. This trend will be probably pursued further in the near future, up to new limits in terms of speed and number of target analytes, so as to manage hundreds of samples per day. Automatically-driven high-resolution MS/MS instruments, sequentially generating data-dependent CID product-ion spectra, will provide true general unknown screening and will allow retrospective inquiry of previously acquired data. Improvements in mass analyzers that will lead to enhanced sensitivity and mass resolution at high scanning rate will 
possibly make these instruments preferable to triple quadrupoles for general unknown screening analysis.

High throughput and costs of analysis are strictly connected to one another. Hair analysis is still an expensive determination that prevents its generalized application to workplace and driving license testing, even if it is evident that drivers and, for example, workers who carry fire-arms should not be habitual drug consumers. The analysis costs are due to the labor-intensive hair-sample treatment and the difficulty to test all drugs of abuse at once. Further development of direct MS methods of analysis, such as DESI or MALDI-TOF, combined with simplified sample handling, could possibly provide very fast and cheap drug screening of hair samples in the future. Even if the sensitivity of these instrumental approaches is still low, quite high cut-off values could be accepted whenever the scope of the screening is to single out only the chronic drug consumers. Also, MSimaging techniques require significant improvement, in terms of hair sample handling and instrumental sensitivity, before generalized application can be found in the chronological assessment of drug intake.

The scientific literature reports no studies on the application of isotope-ratio mass spectrometry (IRMS) to hair analysis to discriminate the natural vs. exogenic origin of substances present therein. The substances of interest might include doping agents, such as steroids, as well as a common rape drug; i.e., gamma-hydroxybutyric acid. The reasons for this lack can be found again in the limited sensitivity of the technique, the restricted sample availability, the complexity of its purification, and that specificity provided by mass selection is lost in the combustion process. The chance of using IRMS to detect the illegal administration of drugs over extended periods of time, as it might occur for androgenic steroids, will possibly push some scientific efforts in this direction in the near future.

In general, the recent major improvements of MS and chromatographic instrumentation have driven forensic toxicology toward previously inconceivable results. Among these studies, it is impressive to note that hair analysis can nowadays provide evidence of a single administration of drugs. The continuous enhancement of MS sensitivity progressively reduces the amount of hair sample needed for the chemical analysis, and extends the chance to investigate newborn children, to detect pre-natal drugs exposure. The scientific goal to gain toxicological information from a single hair has already started, in a worthy competition with forensic genetics. Further unexpected developments of MS instruments are likely to have immediate impact also in the investigations of forensic toxicology, and in particular in hair analysis. 


\section{IV. ABBREVIATIONS}

910 All abbreviations and acronyms used in this article are listed in Table 2. 


\section{REFERENCES}

Agius R, Nadulski N, Kahl HG, Schräder J, Dufaux B, Yegles M, Pragst F. 2010. Validation of a headspace solid-phase microextraction-GC-MS/MS for the determination of ethyl glucuronide in hair according to forensic guidelines. Forensic Sci Int 196:3-9.

Agius R, Nadulski N, Kahl HG, Dufaux B. 2012. Ethyl glucuronide in hair - A highly effective test for the monitoring of alcoholconsumption. Forensic Sci Int 218:10-14.

Albermann ME, Musshoff F, Madea B. 2010. A fully validated high-performance liquid chromatography-tandem mass spectrometry method for the determination of ethyl glucuronide in hair for the proof of strict alcohol abstinence. Anal Bioanal Chem 396:24412447.

Albermann ME, Musshoff F, Madea B. 2011. Comparison of ethyl glucuronide (EtG) and fatty acid ethyl esters (FAEEs) concentrations in hair for testing abstinence. Anal Bioanal Chem 400:175-181.

Albermann ME, Musshoff F, Aengenheister L, Madea B. 2012. Investigations on the influence of different grinding procedures on measured ethyl glucuronide concentrations in hair determined with an optimized and validated LC-MS/MS method. Anal Bioanal Chem 403:769:776.

Aleksa K, Walasek P, Fulga N, Kapur B, Gareri J, Koren G. 2012. Simultaneous detection of seventeen drugs of abuse and metabolites in hair using solid-phase micro extraction (SPME) with GC/MS. Forensic Sci Int 218:31-36.

Álvarez I, Bermejo AM, Tabernero MJ, Fernández P, Cabarcos P, López P. 2009. Microwaveassisted extraction: a simpler and faster method for the determination of ethyl glucuronide in hair by gas chromatography-mass spectrometry. Anal Bioanal Chem 393:1345-1350.

Anielski P. 2008. Hair analysis of anabolic steroids in connection with doping control - results from horse samples. J Mass Spectrom 43: 1001-1008.

Barroso M, Dias M, Vieira DN, Queiroz JA, López-Rivadulla M. 2008. Development and validation of an analytical method for the simultaneous determination of cocaine and its 
main metabolite, benzoylecgonine, in human hair by gas chromatography/mass spectrometry. Rapid Commun Mass Spectrom 22:3320-3326.

Barroso M, Dias M, Vieira DN, López-Rivadulla M, Queiroz JA. 2010a. Simultaneous quantitation of morphine, 6-acetylmorphine, codeine, 6-acetylcodeine and tramadol in hair using mixedmode solid-phase extraction and gas chromatography-mass spectrometry. Anal Bioanal Chem 396:3059-3069.

Barroso M, Costa S, Dias M, Vieira DN, Queiroz JA, Lopez-Rivadulla M. 2010b. Analysis of phenylpiperazine-like stimulants in human hair as trimethylsilyl derivatives by gas chromatography-mass spectrometry. J Chormatogr A. 1217:6274-6280.

Bendroth P, Kronstrand R, Helander A, Greby J, Stephanson N, Krantz P. 2008. Comparison of ethyl glucuronide in hair with phosphatidylethanol in whole blood as post-mortem markers of alcohol abuse. Forensic Sci Int 176:76-81.

Beuck S, Möller I, Thomas A, Klose A, Schlörer N, Schänzer W, Thevis M. 2011. Structure characterisation of urinary metabolites of the cannabimimetic JWH-018 using chemically synthesised reference material for the support of LC-MS/MS-based drug testing. Anal Bional Chem 401:493-505.

Broecker S, Herre S, Wurst B, Zweigenbaum J, Pragst F. 2011. Development practical application of a CID accurate mass spectra library of more than 2500 toxic compounds for systematic toxicological analysis by LC-QTOF-MS with data dependent acquisition. Anal Bioanal Chem 400:101-117.

Broecker S, Herre S, Pragst F. 2012. General unknown screening in hair by liquid chromatographyhybrid quadrupole time-of-flight mass spectrometry (LC-QTOF-MS). Forensic Sci Int 218:68-81.

Bucelli F, Fratini A, Bavazzano P, Comodo N. 2009. Quantification of drugs of abuse and some stimulants in hair samples by liquid chromatography-electrospray ionization ion trap mass spectrometry. J Chromatogr B 877:3931-3936.

Cabarcos P, Hassan HM, Taberneroa MJ, Scott KS. 2012. Analysis of ethyl glucuronide in hair samples by liquid chromatography-electrospray ionization-tandem mass spectrometry (LCESI-MS/MS). J Appl Toxicol doi: 10.1002/jat.1791. 
Caplan YH, Goldberger BA. 2001. Alternative specimens for workplace drug testing. J Anal Toxicol 25:396-399.

Caprara DL, Klein J, Koren G. 2006. Diagnosis of fetal alcohol spectrum disorder (FASD): fatty acid ethyl esters and neonatal hair analysis. Ann Ist Super Sanita 42:39-45.

Chèze M, Duffort G, Deveaux M, Pèpin G. 2005. Hair analysis by liquid chromatography-tandem mass spectrometry in toxicological investigation of drug-facilitated crimes: report of 128 cases over the period June 2003-May 2004 in metropolitan Paris. Forensic Sci Int 153:3-10.

Chèze M, Deveaux M, Martin C, Lhermitte M, Pépin G. 2007. Simultaneous analysis of six amphetamines and analogues in hair, blood and urine by LC-ESI-MS/MS. Application to the determination of MDMA after low ecstasy intake. Forensic Sci Int 170:100-104.

Cooper GA, Kronstrand R, Kintz P. 2012. Society of Hair Testing guidelines for drug testing in hair. Forensic Sci Int 218:20-24.

Cordero R, Paterson S. 2007. Simultaneous quantification of opiates, amphetamines, cocaine and metabolites and diazepam and metabolite in a single hair sample using GC-MS. J Chromatogr B 850:423-431.

Deshmukh N, Hussain I, Barker J, Petroczi A, Naughton DP. 2010. Analysis of anabolic steroids in human hair using LC-MS/MS. Steroids 75: 710-714.

Deshmukh N, Barker J, Petroczi A, Naughton DP. 2012. Detection of testosterone and epitestosterone in human hair using liquid chromatography-tandem mass spectrometry. $\mathbf{J}$ Pharm Biomed Anal doi.org/10.1016/j.jpba.2012.04.011.

Di Corcia D, D’Urso F, Gerace E, Salomone A, Vincenti M. 2012. Simultaneous determination in hair of multi-class drugs of abuse (including THC) by ultra-high performance liquid chromatography-tandem mass spectrometry. J Chromatogr B doi:10.1016/j.jchromb.2012.05.003.

Domínguez-Romero JC, García-Reyes JF, Molina-Díaz A. 2011. Screening and quantitation of multi-class drugs of abuse and pharmaceuticals in hair by fast liquid chromatography electrospray time-of-flight mass spectrometry. J Chromatogr B 879:2034-2042. 
Dresen S, Kneisel S, Weinmann W, Zimmermann R, Auwärter V. 2011. Development and validation of a liquid chromatography-tandem mass spectrometry method for the quantitation of synthetic cannabinoids of the aminoalkylindole type and methanandamide in serum and its application to forensic samples. J Mass Spectrom 46:163-171.

Emídio ES, Prata Vde M, Dórea HS. 2010. Validation of an analytical method for analysis of cannabinoids in hair by headspace solid-phase microextraction and gas chromatography-ion trap tandem mass spectrometry. Anal Chim Acta 670:63-71.

EWDTS (European Workplace Drug Testing Society) Guidelines. 2010. Drug and alcohol testing in hair, collection and analysis. EWDTS, August 2010.

Falcon M, Pichini S, Joya J, Pujadas M, Sanchez A, Vall O, Garcìa Algar O, Luna A, de la Torre R, Rotolo MC, Pellegrini M. 2012. Maternal hair testing for the assessment of fetal exposure to drug of abuse during early pregnancy: Comparison with testing in placental and fetal remains. Forensic Sci Int 218:92-96.

Favretto D, Vogliardi S, Stocchero G, Nalesso A, Tuccia M, Ferrara SD. 2012. High performance liquid chromatography-high resolution mass spectrometry and micropulverized extraction for the quantification of amphetamines, cocaine, opioids, benzodiazepines, antidepressants and hallucinogens in $2.5 \mathrm{mg}$ hair samples. J Chromatography A 1218:6583-6595.

Gaillard Y, Vayssette F, Balland A, Pèpin G. 1999. Gas chromatographic-tandem mass spectrometric determination of anabolic steroids and their esters in hair. Application in doping control and meat quality control. J Chromatogr B. 735 :189-205.

Gaillard Y, Vayssette F, Pèpin G. 2000. Compared interest between hair analysis and urinalysis in doping controls. Results for amphetamines, corticosteroids and anabolic steroids in racing cyclists. Forensic Sci Int 107: 361-379.

Gambelunghe C, Sommavilla M, Ferranti C, Rossi R, Aroni K, Manes N, Bacci M. 2007. Analysis of anabolic steroids in hair by GC/MS/MS. Biomed Chromatogr 21: 369-375.

Gareri J, Appenzeller B, Walasek P, Koren G. 2011. Impact of hair-care products on FAEE hair concentrations in substance abuse monitoring. Anal Bioanal Chem 400:183-188. 
Gaulier JM, Sauvage FL, Pauthier H, Saint-Marcoux F, Marquet P, Lachâtre G. 2008. Identification of acepromazine in hair: an illustration of the difficulties encountered in investigating drugfacilitated crimes. J Forensic Sci 53:755-759.

Gottardo R, Fanigliulo A, Bortolotti F, De Paoli G, Pascali JP, Tagliaro F. 2007. Broad-spectrum toxicological analysis of hair based on capillary zone electrophoresis-time-of-flight spectrometry. J Chromatogr A 1159:190-197.

Gottardo R, Mik`ś1k I, Aturki Z, Sorio D, Seri C, Fanali S, Tagliaro F. 2012. Analysis of drugs of forensic interest with capillary zone electrophoresis/time-of-flight mass spectrometry based on the use of non-volatile buffers. Electrophoresis 33:599-606.

Grigoryev A, Savchuk S, Melnik A, Moskaleva N, Dzhurko J, Ershov M, Nosyrev A, Vedenin A, Izotov B, Zabirova I, Rozhanets V. 2011. Chromatography-mass spectrometry studies on the metabolism of synthetic cannabinoids JWH-018 and JWH-073, psychoactive components of smoking mixtures. J Chromatogr B 879:1126-1136.

Guthery B, Bassindale T, Bassindale A, Pillinger CT, Morgan GH. 2010. Qualitative drug analysis of hair extracts by comprehensive two-dimensional gas chromatography/time-of-flight mass spectrometry. J Chromatogr A 1217:4402-4410.

Harun N, Anderson RA, Cormack PAG. 2010. Analysis of ketamine and norketamine in hair samples using molecularly imprinted solid-phase extraction (MISPE) and liquid chromatography-tandem mass spectrometry (LC-MS/MS). Anal Bioanal Chem 396:24492459.

Hastedt M, Herre S, Pragst F, Rothe M, Hartwig S. 2012. Workplace alcohol testing program by combined use of ethyl glucuronide and fatty acid ethyl esters in hair. Alcohol Alcohol 47:127-132.

Hegstad S, Khiabani HZ, Kristoffersen L, Kunøe N, Lobmaier PP, Christophersen AS. 2008. Drug screening of hair by liquid chromatography-tandem mass spectrometry. J Anal Toxicol 32:364-372.

Huang DK, Liu C, Huang MK, Chien CS. 2009. Simultaneous determination of morphine, codeine, 6-acetylmorphine, cocaine and bemzoylecgonine in hair by liquid 
chromatography/electrospray ionization tandem mass spectrometry. Rapid Commun Mass Spectrom 23:957-962.

Irving RC, Dickson SJ. 2007. The detection of sedatives in hair and nail samples using tandem LCMS-MS. Forensic Sci Int 166:58-67.

Jackson AU, Garcia-Reyes JF, Harper JD, Wiley JS, Molina-Dìaz A, Ouyang Z, Cooks RG. 2010. Analysis of drugs of abuse in biofluids by low temperature plasma (LTP) ionization mass spectrometry. Analyst 135:927-933.

Johansen SS, Dahl-Sørensen R. 2012. A drug rape case involving triazolam detected in hair and urine. Int J Legal Med doi:10.1007/s00414-011-0654-6.

Kerekes I, Yegles M, Grimm U, Wennig R. 2009. Ethyl glucuronide determination: head hair versus non-head hair. Alcohol Alcohol 44:62-66.

Kharbouche H, Sporkert F, Troxler S, Augsburger M, Mangin P, Staub C. 2009. Development and validation of a gas chromatography-negative chemical ionization tandem mass spectrometry method for the determination of ethyl glucuronide in hair and its application to forensic toxicology. J Chromatogr B 877:2337-2343.

Kharbouche H, Steiner N, Morelato M, Staub C, Boutrel B, Mangin P, Sporkert F, Augsburger M. 2010. Influence of ethanol dose and pigmentation on the incorporation of ethyl glucuronide into rat hair. Alcohol 44:507-514.

Kim J, Lee S, In S, Choi H, Chung H. 2011. Validation of a simultaneous analytical method for the detection of 27 benzodiazepines and metabolites and zolpidem in hair using LC-MS/MS and its application to human and rat hair. J Chromatogr B 879:878-886.

King LA, Kicman AT. 2011. A brief history of 'new psychoactive substances'. Drug Test Anal $3: 401-403$.

Kintz P, Cirimele V, Ludes B. 2000. Pharmacological criteria that can affect the detection of doping agents in hair. Forensic Sci Int 107: 325-334.

Kintz P. 2003. Testing for anabolic steroids in hair: a review. Leg Med (Tokyo) 5:S29-S33. 
Kintz P, Villain M, Dumestre-Toulet V, Ludes B. 2005. Drug-facilitated sexual assault and analytical toxicology: the role of LC-MS/MS A case involving zolpidem. J Clin Forensic Med 12:36-41.

Kintz P, Villain M, Cirimele V. 2006. Hair analysis for drug detection. Ther Drug Monit 28:442446.

Kintz P. 2007a. Analytical and practical aspects of drug testing in hair. Boca Raton: CRC Press. Taylor \& Francis 382 p.

Kintz P. 2007b. Bioanalytical procedures for detection of chemical agents in hair in the case of drug-facilitated crimes. Anal Bioanal Chem 388:1467-1474.

Kintz P, Villain M, Vallet E, Etter M, Salquebre G, Cirimele V. 2008. Ethyl glucuronide: unusual distribution between head hair and pubic hair. Forensic Sci Int 176:87-90.

Kintz P, Evans J, Villain M, Chatterton C, Cirimele V. 2009. Hair analysis to demonstrate administration of sildenafil to a woman in a case of drug-facilitated sexual assault. J Anal Toxicol 33:553-556.

Kintz P. 2012a. Value of the concept of minimal detectable dosage in human hair. Forensic Sci Int 218:28-30.

Kintz P. 2012b. Consensus of the Society of Hair Testing on hair testing for chronic excessive alcohol consumption 2011. Forensic Sci Int 218:2.

Kłis M, Rojek S, Kulikowska J, Bożek E, Ścisłowski M. 2007. Usefulness of multi-parameter opiates-amphetamines-cocainics analysis in hair of drug users for the evaluation of an abuse profile by means of LC-APCI-MS-MS. J Chromatogr B 854:299-307.

Kraemer T, Rust KY, Meyer MR, Wissenbach DK, Bregel D, Hopf M, Maurer HH, Wilske J. 2009. Studies on the metabolism of JWH-018 and of a homologue of CP 47,497, pharmacologically active ingredients of different misused incense ("spice") using GC-MS and LCMSn techniques. Ann Toxicol Anal 21:S1-S21.

Kronstrand R, Brinkhagen L, Nyström FH. 2012. Ethyl glucuronide in human hair after daily consumption of 16 or $32 \mathrm{~g}$ of ethanol for 3 months. Forensic Sci Int 215:51-55. 
Kulaga V, Caprara D, Iqbal Q, Kapur B, Klein J, Reynolds J, Brien J, Koren G. 2006. Fatty acid ethyl esters (FAEE); comparative accumulation in human and guinea pig hair as a biomarker for prenatal alcohol exposure. Alcohol Alcohol 41:534-539.

Kulaga V, Velazquez-Armenta Y, Aleksa K, Vergee Z, Koren G. 2009. The effect of hair pigment on the incorporation of fatty acid ethyl esters (FAEE). Alcohol Alcohol 44:287-292.

Laloup M, Ramirez Fernandez MM, Wood M, Maes V, De Boeck G, Vanbeckevoort, Samyn N. 2007. Detection of diazepam in urine, hair and preserved oral fluid samples with LC-MSMS after single and repeated administration of Myolastan and Valium. Anal Bioanal Chem 388:1545-1556.

Lamoureux F, Gaulier JM, Sauvage FL, Mercerolle M, Vallejo C, Lachâtre G. 2009. Determination of ethyl-glucuronide in hair for heavy drinking detection using liquid chromatographytandem mass spectrometry following solid-phase extraction. Anal Bioanal Chem 394:18951901.

LeBeau MA, Mozayani A. 2001. Collection of evidence from DFSA. In: LeBeau MA, Mozayani A, Editor. Drug-Facilitated Sexual Assault: a forensic handbook. San Diego: Academic Press. p 197-209.

LeBeau MA, Montgomery MA. 2009. Considerations on the utility of hair analysis for cocaine. J Anal Toxicol 33: 343-344.

LeBeau MA, Montgomery MA. 2010. Challenges of Drug-Facilitated Sexual Assault. Forensic Sci Rev 22:1-6.

Lee S, Han E, Park Y, Choi H, Chung H. 2009. Distribution of methamphetamine and amphetamine in drug abusers' head hair. Forensic Sci Int 190:16-18.

Lee S, Kim J, In S, Choi H, Oh SM, Jang CG, Chung KH. 2012. Development of a simultaneous analytical method for selected anorectics, methamphetamine, MDMA, and their metabolites in hair using LC-MS/MS to prove anorectics abuse. Anal Bioanal Chem 403:1385-1394.

Lendoiro E, Quintela O, de Castro A., Cruz A, Lopez-Rivadulla M, Concheiro M. 2012. Target screening and confirmation of 35 licit and illicit drugs and metabolites by LC-MSMS. Forensic Sci Int 217:207-215. 
Lin YH, Lee MR, Lee RJ, Ko WK, Wu SM. 2007. Hair analysis for metamphetamine, ketamine, morphine and codeine by cation-selective exhaustive injection and sweeping electrokinetic chromatography. J. Chromatrogr. A 1145:234-240.

Liu HC, Liu RH, Lin DL, Ho HO. 2010. Rapid screening and confirmation of drugs and toxic compounds in biological specimens using liquid chromatography/ion trap tandem mass spectrometry and automated library search. Rapid Commun Mass Spectr 24:75-84.

Marques P, Tippetts S, Allen J, Javors M, Alling C, Yegles M, Pragst F, Wurst F. 2010. Estimating driver risk using alcohol biomarkers, interlock blood alcohol concentration tests and psychometric assessments: initial descriptives. Addiction 105:226-239.

Martin M, Muller JF, Turner K, Duez M, Cirimele V. 2012. Evidence of mephedrone chronic abuse through hair analysis using GC/MS. Forensic Sci Int 218:44-48.

Matuszewski BK, Constanzer ML, Chavez-Eng CM. 2003. Strategies for the assessment of matrix effect in quantitative bioanalytical methods based on HPLC-MS/MS. Anal Chem 75:30193030 .

Merola G, Gentili S, Tagliaro F, Macchia T. 2010. Determination of different recreational drugs in hair by HS-SPME and GC/MS. Anal Bioanal Chem 397:2987:2995.

Miki A, Katagi M, Kamata T, Zaitsu K, Tatsuno M, Nakanishi T, Tsuchihashi H, Takubo T, Suzuki K. 2011. MALDI-TOF and MALDI-FTICR imaging mass spectrometry of methamphetamine incorporated into hair. J Mass Spectrom 46:411-416.

Miller EI, Wylie FM, Oliver JS. 2008. Simultaneous detection and quantification of amphetamines, diazepam and its metabolites, cocaine and its metabolites, and opiates in hair by LC-ESIMS-MS using a single extraction method. J Anal Toxicol 32:457-469.

Miyaguchi H, Kakuta M, Iwata YT, Matsuda H, Tazawa H, Kimura H, Inoue H. 2007. Development of a micropulverized extraction method for rapid toxicological analysis of methamphetamine in hair. J Chormatogr A 1163:43-48.

Miyaguchi H, Iwata YT, Kanamori T, Tsujikawa K, Kuwayama K, Inoue H. 2009. Rapid identification and quantification of methamphetamine and amphetamine in hair by gas 
chromatography/mass spectrometry coupled with micropulverized extraction, aqueous acetylation and microextraction by packed sorbent. J Chormatogr A 1216:4063-4070.

Moore C, Coulter C, Crompton K. 2007. Determination of cocaine, benzoylecgonine, cocaethylene and norcocaine in human hair using solid-phase extraction and liquid chromatography with tandem mass spectrometric detection. J Chromatogr B 859:208-212.

Moran CL, Le VH, Chimalakonda KC, Smedley AL, Lackey FD, Owen SN, Kennedy PD, Endres GW, Ciske FL, Kramer JB, Kornilov AM, Bratton LD, Dobrowolski PJ, Wessinger WD, Fantegrossi WE, Prather PL, James LP, Radominska-Pandya A, Moran JH. 2011. Quantitative Measurement of JWH-018 and JWH-073 Metabolites Excreted in Human Urine. Anal Chem 83:4228-4236.

Morini L, Politi L, Polettini A. 2009. Ethyl glucuronide in hair. A sensitive and specific marker of chronic heavy drinking. Addiction 104:915-920.

Morini L, Zucchella A, Polettini P, Politi L, Groppi A. 2010. Effect of bleaching on ethyl glucuronide in hair: an in vitro experiment. Forensic Sci Int 198:23-27.

Nadulski T, Pragst F. 2007. Simple and sensitive determination of Delta(9)-tetrahydrocannabinol, cannabidiol and cannabinol in hair by combined silylation, headspace solid-phase microextraction and gas chromatography-mass spectrometry. J Chromatogr B 846:78-85.

Negrusz A, Moore CM, Kern JL, Janicak PG, Strong MJ, Levy NA. 2000. Quantitation of clonazepam and its major metabolite 7-aminoclonazepam in hair. J Anal Toxicol 24:614620.

Negrusz A, Moore CM, Hinkel KB, Stockham TL, Verma M, Strong MJ, Janicak PG. 2001. Deposition of 7-aminoflunitrazepam and flunitrazepam in hair after a single dose of Rohypnol. J Forensic Sci 46:1143-1151.

Negrusz A, Gaensslen RE. 2003. Analytical developments in toxicological investigation of drugfacilitated sexual assault. Anal Bioanal Chem 376:1192-1197.

Nielen MWF, Nijroldera AWJM, Hooijerinka H, Stolkera AAM. 2011. Feasibility of desorption electrospray ionization mass spectrometry for rapid screening of anabolic steroid esters in hair. Anal Chim Acta 700:63-69. 
Nielsen MK, Johansen SS, Dalsgaard PW, Linnet K. 2010. Simultaneous screening and quantification of 52 common pharmaceuticals and drugs of abuse in hair using UPLC-TOFMS. Forensic Sci Int 196:85-92.

Niessen WMA. 2011. Fragmentation of toxicologically relevant drugs in positive-ion liquid chromatography-tandem mass spectrometry. Mass Spectrom Rev 30:626-663.

Parkin MC, Brailsford AD. 2009. Retrospective drug detection in cases of drug-facilitated sexual assault: challenges and perspectives for the forensic toxicologist. Bioanalysis 1:1001-1013.

Paul R, Kingston R, Tsanaclis L, Berry A, Guwy A. 2008. Do drug users use less alcohol than nondrug users? A comparison of ethyl glucuronide concentrations in hair between the two groups in medico-legal cases. Forensic Sci Int 176:82-86.

Paul R, Tsanaclis L, Kingston R, Berry A, Guwy A. 2011. Simultaneous determination of GHB and EtG in hair using GCMS/MS. Drug Test Anal 3:201-205.

Pelander A, Ristimaa J, Rasanen I, Vuori E, Ojanperä I. 2008. Screening for basic drugs in hair of drug addicts by liquid chromatography/time-of-flight mass spectrometry. Ther Drug Monit 30:717-724.

Peters FT, Remane D. 2012. Aspects of matrix effects in applications of liquid chromatographymass spectrometry to forensic and clinical toxicology-a review. Anal Bioanal Chem doi: 10.1007/s00216-012-6035-2.

Pirro V, Valente V, Oliveri P, De Bernardis A, Salomone A, Vincenti M. 2011a. Chemometric evaluation of nine alcohol biomarkers in a large population of clinically-classified subjects: pre-eminence of ethyl glucuronide concentration in hair for confirmatory classification. Anal Bioanal Chem 401:2153-2164.

Pirro V, Di Corcia D, Pellegrino S, Vincenti M, Sciutteri B, Salomone A. 2011b. A study of distribution of ethyl glucuronide in different keratin matrices. Forensic Sci Int 210:271-277.

Polettini A, Cone EJ, Gorelick DA, Huestis MA. 2012. Incorporation of methamphetamine and amphetamine in human hair following controlled oral methamphetamine administration. Anal Chim Acta 726:35-43. 
Politi L, Morini L, Leone F, Polettini A. 2006. Ethyl glucuronide in hair: is it a reliable marker of chronic high levels of alcohol consumption?. Addiction 101:1408-1412.

Politi L, Morini L, Mari F, Groppi A, Bertol E. 2008. Ethyl glucuronide and ethyl sulfate in autopsy samples 27 years after death. Int J Leg Med 122:507-509.

Politi L, Mari F, Furlanetto S, Del Bravo E, Bertol E. 2011. Determination of fatty acid ethyl esters in hair by GC-MS and application in a population of cocaine users. J Pharm Biomed Anal 54:1192-1195.

Porta T, Grivet C, Kraemer T, Varesio E, Hopfgartner G. 2011. Single hair cocaine consumption monitoring by mass spectrometry imaging. Anal Chem 83:4266-4272.

Pozo OJ, Deventer K, Van Eenoo P, Rubens R, Delbeke FT. 2009. Quantification of testosterone undecanoate in human hair by liquid chromatography-tandem mass spectrometry. Biomed Chromatogr 23: 873-880.

Pragst F, Auwaerter V, Sporkert F, Spiegel K. 2001. Analysis of fatty acid ethyl esters in hair as possible markers of chronically elevated alcohol consumption by headspace solid-phase microextraction (HS-SPME) and gas chromatography-mass spectrometry (GC-MS). Forensic Sci Int 121:76-88.

Pragst F, Nadulski T. 2005. Limite de détection du THC dans les cheveux dans le contexte de l'aptitude à la conduite. Ann Toxicol Anal 17:237-240.

Pragst F, Balikova MA. 2006. State of the art in hair analysis for detection of drug and alcohol abuse. Clin Chim Acta 370:17-49.

Pragst F, Yegles M. 2008. Determination of fatty acid ethyl esters (FAEE) and ethyl glucuronide (EtG) in hair: a promising way for retrospective detection of alcohol abuse during pregnancy?. Ther Drug Monit 30:255-263.

Pragst F, Rothe M, Moench B, Hastedt M, Herre S, Simmert D. 2010. Combined use of fatty acid ethyl esters and ethyl glucuronide in hair for diagnosis of alcohol abuse: interpretation and advantages. Forensic Sci Int 196:101-110. 
Quintela O, Lendoiro E, Cruz A, de Castro A, Quevedo A, Jurado C, López-Rivadulla M. 2010. Hydrophilic interaction liquid chromatography-tandem mass spectrometry (HILIC-MS/MS) determination of cocaine and its metabolites benzoylecgonine, ecgonine methyl ester, and cocaethylene in hair samples. Anal Bioanal Chem 396:1703-1712.

Rodrigues de Oliveira CD, Yonamine M, and de Moraes Moreau RL 2007. Headspace solid-phase microextraction of cannabinoids in human head hair samples. J Sep Sci 30:128-134.

Salomone A, Gerace E, Di Corcia D, Martra G, Petrarulo M, Vincenti M. 2012a. Hair analysis of drugs involved in drug-facilitated sexual assault and detection of zolpidem in a suspected case. Int J Legal Med 126:451-459.

Salomone A, Gerace E, D’Urso F, Di Corcia D, Vincenti M. 2012b. Simultaneous analysis of several synthetic cannabinoids, THC, CBD, CBN in hair by ultra-high performance liquid chromatography tandem mass spectrometry. Method validation and application to real samples. J Mass Spectrom 47:604-610.

Scott KS. 2009. The use of hair as a toxicological tool in DFC casework. Sci Justice 49:250-253.

Shen M, Xiang P, Shen B, Bu J, Wang M. 2009. Physiological concentrations of anabolic steroids in human hair. Forensic Sci Int 184: 32-36.

Shi Y, Shen B, Xiang P, Yan Y, Shen M. 2010. Determination of ethyl glucuronide in hair samples of Chinese people by protein precipitation (PPT) and large volume injection-gas chromatography-tandem mass spectrometry (LVI-GC/MS/MS). J Chromatogr B 878:31613166.

Sobolevsky T, Prasolov I, Rodchenkov G. 2010. Detection of JWH-018 metabolites in smoking mixture post-administration urine. Forensic Sci Int 200:141-147.

Sporkert F, Kharbouche H, Augsburger MP, Klemm C, Baumgartner MR. 2012. Positive EtG findings in hair as a result of a cosmetic treatment. Forensic Sci Int 218:97-100.

Stout PA, Simons KD, Kerrigan S. 2010. Quantitative analysis of gamma-hydroxybutyrate at endogenous concentrations in hair using liquid chromatography tandem mass spectrometry. J Forensic Sci 55:531-537. 
Süsse S, Selavka CM, Mieczkowski T, Pragst F. 2010. Fatty acid ethyl ester concentrations in hair and self-reported alcohol consumption in 644 cases from different origin. Forensic Sci Int 196:111-117.

Süsse S, Pragst F, Mieczkowski T, Selavka CM, Elian A, Sachs H, Hastedt M, Rothe M, Campbell J. 2012. Practical experiences in application of hair fatty acid ethyl esters and ethyl glucuronide for detection of chronic alcohol abuse in forensic cases. Forensic Sci Int 218:82-91.

Tagliaro F, Bortolotti F, Viel G, Ferrara SD. 2011. Caveats against an improper use of hair testing to support the diagnosis of chronic excessive alcohol consumption, following the "Consensus" of the Society of Hair Testing 2009 [Forensic Science International 196 (2010) 2]. Forensic Sci Int 207:e69-e70.

Takáts Z, Wiseman JM, Gologan B, Cooks RG. 2004. Mass spectrometry sampling under ambient conditions with desorption electrospray ionization. Science 306:471-473.

Tarcomnicu I, van Nuijs AL, Aerts K, De Doncker M, Covaci A, Neels H. 2010. Ethyl glucuronide determination in meconium and hair by hydrophilic interaction liquid chromatographytandem mass spectrometry. Forensic Sci Int 196:121-127.

Teske J, Weller JP, Fieguth A, Rothämel T, Schulz Y, Tröger HD. 2010. Sensitive and rapid quantification of the cannabinoid receptor agonist naphthalen-1-yl-(1-pentylindol-3-yl) methanone (JWH-018) in human serum by liquid chromatography-tandem mass spectrometry. J Chromatogr B 878:2959-2963.

Thibert V, Legeay P, Chapuis-Hugon F, Pichon V. 2012. Synthesis and characterization of molecularly imprinted polymers for the selective extraction of cocaine and its metabolite benzoylecgonine from hair extract before LC-MS analysis. Talanta 88:412:419.

Thieme D, Grosse J, Sachs H, Mueller RK. 2000. Analytical strategy for detecting doping agents in hair. Forensic Sci Int 107: 335-345.

Thieme D, Sachs H. 2007. Examination of a long-term clozapine administration by high resolution segmental hair analysis. Forensic Sci Int 166:110-114. 
Torrance H, Cooper G. 2010. The detection of mephedrone (4-methylmethcathinone) in 4 fatalities in Scotland. Forensic Sci Int 202:e62-e63.

UNODC (United Nations Office on Drug and Crime). 2011. Guidelines for the Forensic analysis of drugs facilitating sexual assault and other criminal acts. UNODC, December 2011.

Villain M, Chèze M, Tracqui A, Ludes B, Kintz P. 2004. Windows of detection of zolpidem in urine and hair: application to two drug facilitated sexual assaults. Forensic Sci Int 143:157161.

Villain M, Concheiro M, Cirimele V, Kintz P. 2005. Screening method for benzodiazepines and hypnotics in hair at pg/mg level by liquid chromatography-mass spectrometry/mass spectrometry. J Chromatogr B 825:72-78.

Vogliardi S, Favretto D, Frison G, Ferrara SD, Seraglia R, Traldi P. 2009. A fast screening MALDI method for the detection of cocaine and its metabolites in hair. J Mass Spectrom 44:18-24.

Vogliardi S, Favretto D, Frison G, Maietti S, Viel G, Seraglia R, Traldi P, Ferrara SD. 2010. Validation of a fast screening method for the detection of cocaine in hair by MALDI-MS. Anal Bioanal Chem 396:2435-2440.

Vogliardi S, Favretto D, Tucci M, Stocchero G, Ferrara SD. 2011. Simultaneous LC-HRMS determination of 28 benzodiazepines and metabolites in hair. Anal Bioanal Chem 400:51-67.

Wada M, Ikeda R, Kuroda N, Nakashima K. 2010. Analytical methods for abused drugs in hair and their applications. Anal Bioanal Chem 397:1039-1067.

WADA (World Anti-Doping Agency). 2012. International standard for laboratories, Version 7.0. WADA, January 2012.

Wintermeyer A, Möller I, Thevis M, Jübner M, Beike J, Rothschild MA, Bender K. 2010. In vitro phase I metabolism of the synthetic cannabimimetic JWH-018. Anal Bional Chem 398:2141-2153.

Wu YH, Lin KL, Chen SC, Chang YZ. 2008a. Simultaneous quantitative determination of amphetamines, ketamine, opiates and metabolites in human hair by gas chromatography/mass spectrometry. Rapid Commun Mass Spectrom 22:887-897. 
Wu YH, Lin KL, Chen SC, Chang YZ. 2008b. Integration of GC/EI-MS and GC/NCI-MS for simultaneous quantitative determination of opiates, amphetamines, MDMA, ketamine, and metabolites in human hair. J Chromatogr B 870:192-202.

Xiang P, Sun Q, Shen B, Chen P, Liu W, Shen M. 2011. Segmental hair analysis using liquid chromatography-tandem mass spectrometry after a single dose of benzodiazepines. Forensic Sci Int 204:19-26.

Zimmermann CM, Jackson GP. 2010. Gas chromatography tandem mass spectrometry for biomarkers of alcohol abuse in human hair. Ther Drug Monit 32:216-223. 
TABLE 1. Comparison of MS conditions for EtG determination in hair samples

\begin{tabular}{|c|c|c|c|c|c|}
\hline Authors, year & $\begin{array}{l}\text { Analytical } \\
\text { Technique }\end{array}$ & Mass Analyzer & \multicolumn{2}{|c|}{$\begin{array}{c}m / z \text { Ions } \\
\text { (acquisition mode) }\end{array}$} & Derivatization \\
\hline Álvarez et al., 2009 & GC-EI-MS & single quadrupole & $261 ; 160 ; 405$ & (SIM) & BSTFA \\
\hline $\begin{array}{l}\text { Paul et al., } 2008 \\
\text { Paul et al., } 2011\end{array}$ & GC-EI-MS/MS & triple quadrupole & $261 \rightarrow 143$ & $(\mathrm{SRM})$ & BSTFA \\
\hline Shi et al., 2010 & GC-EI-MS/MS & triple quadrupole & $\begin{array}{c}261 \rightarrow 143 \\
261 \rightarrow 73\end{array}$ & $(\mathrm{SRM})$ & BSTFA \\
\hline $\begin{array}{l}\text { Kharbouche et al., } 2009 \\
\text { Kharbouche et al., } 2010 \\
\text { Sporkert et al., } 2012\end{array}$ & GC-NCI-MS/MS & triple quadrupole & $\begin{array}{l}347 \rightarrow 163 \\
347 \rightarrow 119\end{array}$ & (SRM) & PFPA \\
\hline Agius et al., 2010 & GC-NCI-MS/MS & triple quadrupole & $\begin{array}{l}596 \rightarrow 427 \\
596 \rightarrow 288\end{array}$ & (SRM) & HFBA \\
\hline Kerekes et al., 2009 & GC-NCI-MS & single quadrupole & $496 ; 349$ & (SIM) & PFPA \\
\hline $\begin{array}{l}\text { Morini et al., } 2009 \\
\text { Morini et al., } 2010 \\
\text { Politi et al., } 2008\end{array}$ & LC-ESI-MS/MS & triple quadrupole & $\begin{array}{l}221 \rightarrow 221 \\
221 \rightarrow 85 \\
221 \rightarrow 75\end{array}$ & $(\mathrm{SRM})$ & - \\
\hline $\begin{array}{c}\text { Pirro et al., 2011a } \\
\text { Pirro et al., 2011b } \\
\text { Kronstrand et al., } 2012 \\
\text { Pragst et al., } 2010 \\
\text { Hastedt et al., } 2012 \\
\text { Lamoreux et al., } 2009 \\
\text { Tarcumnicu et al., } 2010 \\
\text { Kintz et al., 2008 }\end{array}$ & LC-ESI-MS/MS & triple quadrupole & $\begin{aligned} 221 & \rightarrow 85 \\
221 & \rightarrow 75\end{aligned}$ & $(\mathrm{SRM})$ & - \\
\hline $\begin{array}{l}\text { Süsse et al., } 2010 \\
\text { Süsse et al., } 2012\end{array}$ & LC-ESI-MS/MS & triple quadrupole & $\begin{array}{l}221 \rightarrow 75 \\
221 \rightarrow 57 \\
221 \rightarrow 55\end{array}$ & (SRM) & - \\
\hline Cabarcos et al., 2012 & LC-ESI-MS/MS & ion trap & $\begin{array}{l}221 \rightarrow 203 \\
221 \rightarrow 85 \\
221 \rightarrow 75\end{array}$ & (SRM) & - \\
\hline $\begin{array}{l}\text { Albermann et al., } 2010 \\
\text { Albermann et al., } 2012\end{array}$ & LC-ESI-MS/MS & triple quadrupole & $\begin{aligned} 221 & \rightarrow 113 \\
221 & \rightarrow 85 \\
221 & \rightarrow 75\end{aligned}$ & (SRM) & - \\
\hline
\end{tabular}


TABLE 2. Abbreviations and acronyms used in this article.

\begin{tabular}{|c|c|}
\hline 6-MAM & 6-monoacetylmorphine \\
\hline AASs & Anabolic Androgenic Steroids \\
\hline AP & Amphetamine \\
\hline APCI & Atmospheric Pressure Chemical Ionization \\
\hline BSTFA & N,O-bis[trimethylsilyl]trifluoroacetamide \\
\hline $\mathrm{BZE}$ & Benzoylecgonine \\
\hline $\mathrm{CBN}$ & Cannabinol \\
\hline $\mathrm{CDB}$ & Cannabidiol \\
\hline CDT & Carbohydrate-Deficient Transferrin \\
\hline $\mathrm{CI}$ & Chemical Ionization \\
\hline CID & Collision-Induced Dissociation \\
\hline CNS & Central Nervous System \\
\hline $\mathrm{CZE}$ & Capillary Zone Electrophoresis \\
\hline DESI & Desorption Ionization Mass Spectrometry \\
\hline DFC & Drug Facilitated Crime \\
\hline DFSA & Drug Facilitated Sexual Assault \\
\hline DHEA & Dehydroepiandrosterone \\
\hline E12 & Ethyl laureate \\
\hline E14 & Ethyl myristate \\
\hline E16 & Ethyl palmitate \\
\hline E16:1 & Ethyl palmitoleate \\
\hline E18 & Ethyl stearate \\
\hline E18:1 & Ethyl oleate \\
\hline EI & Electron Impact \\
\hline ELISA & Enzyme-Linked ImmunoSorbent Assay \\
\hline EME & Ecgonine Methyl Ester \\
\hline ESI & Electrospray Ionization \\
\hline EtG & Ethyl Glucuronide \\
\hline EtS & Ethyl Sulfate \\
\hline FAEE & Fatty Acid Ethyl Esters \\
\hline FTICR & Fourier Transform Ion Cyclotron Resonance \\
\hline GC & Gas Chromatography \\
\hline HFBA & Heptafluorobutyric Anhydride \\
\hline HILIC & Hydrophilic Interaction Liquid Chromatography \\
\hline HPLC & High Pressure Liquid Chromatography \\
\hline HRMS & High Resolution Mass Spectrometry \\
\hline HS-SPME & Head Space Solid-phase MicroEstraction \\
\hline IMS & Imaging Mass Spectrometry \\
\hline IRMS & Isotope-Ratio Mass Spectrometry \\
\hline $\mathrm{LC}$ & Liquid Chromatography \\
\hline LLE & Liquid-Liquid Extraction \\
\hline LOD & Limit of Detection \\
\hline LOQ & Limit of Quantification \\
\hline
\end{tabular}


TABLE 2. (Continued)

\begin{tabular}{|c|c|}
\hline LVI & Large Volume Injection \\
\hline LTP & Low Temperature Plasma \\
\hline MALDI & Matrix-Assisted Laser Desorption Ionization \\
\hline MBDB & N-methyl-1-(3,4-methylenedioxyphenyl)-2-butanamine \\
\hline MBTFA & N-methyl-bis trifluoroacetamide \\
\hline MDA & 3,4-methylenedioxyamphetamine \\
\hline MDEA & 3,4-methylenedioxyethamphetamine \\
\hline MDMA & 3,4-methylenedioxymethamphetamine \\
\hline MiAMi & Micropulverized Extraction Aqueous Acetylation Microextraction \\
\hline MIP & Molecularly Imprinted Polimers \\
\hline MISPE & Molecularly Imprinted Solid-Phase MicroExtraction \\
\hline MS & Mass Spectrometry \\
\hline MSTFA & $\mathrm{N}$-methyl-N-trimethylsilyltrifluoroacetamide \\
\hline MTBSTFA & N-methyl-N-(tert-butyldimethyl)trifluoroacetamide \\
\hline NCI & Negative Chemical Ionization \\
\hline PEth & Phosphatidylethanol \\
\hline PTV & Programmable Temperature Vaporization \\
\hline QqQ & Triple quadrupole mass spectrometer \\
\hline QqQLIT & Hybrid triple quadrupole linear ion trap mass spectrometer \\
\hline QuEChERS & Quick Easy Cheap Effective Rugged Safe \\
\hline SIM & Selected Ion Monitoring \\
\hline SOHT & Society of Hair Testing \\
\hline SOHT & Society of Hair Testing \\
\hline SPE & Solid-phase Extraction \\
\hline SRM & Selected Reaction Monitoring \\
\hline THC & $\Delta^{9}$-tetrahydrocannabinol \\
\hline TMCS & Trimethylchlorosilane \\
\hline TOF & Time of Flight \\
\hline UHPLC & Ultra High Performance Liquid Chromatography \\
\hline WADA & World Anti-Doping Agency \\
\hline
\end{tabular}




\section{FIGURE CAPTIONS}

FIGURE 1. MALDI-MS analytical workflow for single hair analysis. The whole process from sample preparation to SRM relative quantitation and MS/MS and $\mathrm{MS}^{3}$ confirmatory analyses takes approximately $2 \mathrm{~h}$, including the analysis of multiple samples (Porta et al., 2011; reproduced with permission, American Chemical Society, copyright 2011).

FIGURE 2. GC $\times \mathrm{GC}$ contour plot (TIC) obtained for a hair sample. (1) Cotinine; (2) meconin; (3) mesocain; (4) tropacaine; 5.EDDP; (6) phenazocine; (7) hydroxycotinine-TBDMS; (8) methadone; (9) quaalude; (10) norcocaine; (11) cocaine; (12) cocaethylene; (13) codeine; (14) diazepam; (15) benzoylecgonine-TBDMS; (16) acetylcodeine; (17) cinnamoylcocaine; (18) diacetylmorphine; (19) codeine-TBDMS; (20) morphine-TBDMS; (21) 6-MAM-TBDMS; (22) Papaverine; (23) morphinedi-TBDMS (Guthery et al., 2010; reproduced with permission, Elsevier, copyright 2010).

FIGURE 3. Concentration of head hair EtG (pg/mg) versus \%CDT for 175 patients clinically classified as non-drinkers ( $A$, green dots, $\mathrm{N}=65$ ), social drinkers ( $\mathrm{S}$, yellow dots, $\mathrm{N}=51$ ), and active heavy drinkers $(\mathrm{H}$, red dots, $\mathrm{N}=59)$. The data are reported on a logarithmic scale. Horizontal and vertical lines divide the plane into four quadrants (I-IV) that identify the number of true and false positive (or negative) results in each category: For heavy drinkers (red dots): I. True-positive results; II. false-negative results for CDT only; III. false-negative results; IV. false-negative results for head hair EtG only (Pirro et al., 2011; redrawn with permission, Springer, copyright 2011).

FIGURE 4. Proposed fragmentation pattern for testosterone undecanoate (Pozo et al., 2009; reproduced with permission, John Wiley and Sons, copyright 2009).

FIGURE 5. SRM chromatogram of a blank hair sample fortified with the target compounds: (1) JWH-200, (2) JWH-250, (3) JWH-073, (4) CBD, (5) JWH-018, (6) CBN, (7) HU-210, (8) THC, (ISTD) JWH-018- $d 9$ (Salomone et al., 2012; reproduced with permission, John Wiley and Sons, copyright 2012). 